ARTICLE

\title{
YAP and TAZ protect against white adipocyte cell death during obesity
}

\author{
Lei Wang (10 ${ }^{1 凶}$, ShengPeng Wang (10 ${ }^{1,2 凶}$, Yue Shi ${ }^{2}$, Rui Li ${ }^{1}$, Stefan Günther ${ }^{3}$, Yu Ting Ong (i) ${ }^{4}$, Michael Potente ${ }^{4}$, \\ Zuyi Yuan ${ }^{5}$, Enqi Liu ${ }^{6} \&$ Stefan Offermanns (i) ${ }^{1,7 凶}$
}

The expansion of the white adipose tissue (WAT) in obesity goes along with increased mechanical, metabolic and inflammatory stress. How adipocytes resist this stress is still poorly understood. Both in human and mouse adipocytes, the transcriptional co-activators YAP/TAZ and YAP/TAZ target genes become activated during obesity. When fed a high-fat diet (HFD), mice lacking YAP/TAZ in white adipocytes develop severe lipodystrophy with adipocyte cell death. The pro-apoptotic factor BIM, which is downregulated in adipocytes of obese mice and humans, is strongly upregulated in YAP/TAZ-deficient adipocytes under $\mathrm{HFD}$, and suppression of BIM expression reduces adipocyte apoptosis. In differentiated adipocytes, TNF $\alpha$ and IL-1 $\beta$ promote YAP/TAZ nuclear translocation via activation of RhoAmediated actomyosin contractility and increase YAP/TAZ-mediated transcriptional regulation by activation of c-Jun N-terminal kinase (JNK) and AP-1. Our data indicate that the YAP/ TAZ signaling pathway may be a target to control adipocyte cell death and compensatory adipogenesis during obesity.

\footnotetext{
${ }^{1}$ Department of Pharmacology, Max Planck Institute for Heart and Lung Research, Bad Nauheim 61231, Germany. ${ }^{2}$ Cardiovascular Research Center, School of Basic Medical Sciences, Xi'an Jiaotong University Health Science Center, Yanta District, Xi'an, China. ${ }^{3}$ Bioinformatics and Deep Sequencing Platform, Max Planck Institute for Heart and Lung Research, Bad Nauheim 61231, Germany. ${ }^{4}$ Angiogenesis and Metabolism Laboratory, Max Planck Institute for Heart and Lung Research, Bad Nauheim 61231, Germany. ${ }^{5}$ Department of Cardiology, First Affiliated Hospital of Xi'an Jiaotong University, Xi'an, China. ${ }^{6}$ Laboratory Animal Center, Xi'an Jiaotong University Health Science Center Xi'an Jiaotong University, Xi'an, China. ${ }^{7}$ Center for Molecular Medicine, Medical Faculty, Goethe

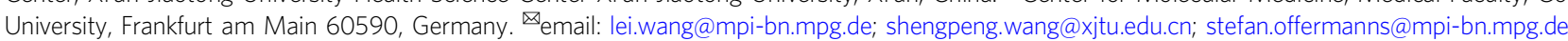


O besity is a serious health problem as it is a strong risk factor for the development of type- 2 diabetes, cardiovascular diseases, and cancer ${ }^{1-6}$. In obesity, the adipose tissue undergoes dynamic remodeling including an increase in size (hypertrophy) and number of adipocytes (hyperplasia), an infiltration by immune cells, and the development of tissue fibrosis $^{7-12}$. In the obese adipose tissue, adipocytes are exposed to different forms of stress. This includes hypoxia, oxidative stress, metabolic stress, mechanical stress from hypertrophy, and inflammatory stress due to infiltration of the adipose tissue by immune cells and increased levels of cytokines ${ }^{13-19}$. Different forms of cellular stress can activate extrinsic and intrinsic pathways of cell death resulting in adipocyte apoptosis ${ }^{20-23}$. There are also anti-apoptotic, pro-survival pathways activated in adipocytes of the obese adipose tissue, which protect adipocytes against cell death. This is indicated by various mouse models with defects in pro-survival pathways, which show strongly increased adipocyte death during obesity ${ }^{24-26}$. However, the mechanisms protecting adipocytes from uncontrolled cell death during obesity are still poorly understood.

YAP and its paralog TAZ are transcriptional cofactors, which regulate various cell functions including cell proliferation, differentiation, and cell survival by integrating different cellular signaling pathways ${ }^{27-30}$. Their nuclear translocation and activity are controlled by several upstream signaling pathways. Activation of the Hippo pathway results in phosphorylation of YAP/TAZ by LATS kinase, leading to the cytoplasmic retention and degradation of YAP/TAZ ${ }^{30-32}$. In addition, G-protein-mediated signaling pathways involving $\mathrm{G}_{12} / \mathrm{G}_{13}$ or $\mathrm{G}_{\mathrm{q}} / \mathrm{G}_{11} 33,34$, as well as extracellular matrix stiffness and cytoskeletal tension, can promote nuclear translocation and activation of YAP/TAZ $28,35-37$. Nuclear YAP/TAZ can co-activate or co-repress numerous genes involved in cell proliferation, differentiation, and survival by interacting with different transcription factors of which the TEAD (TEA domain) family transcription factors are the best characterized $^{32,38-40}$. Given that YAP and TAZ play vital functions in cell survival, we hypothesized that YAP and TAZ are crucial for adipocyte homeostasis.

Here we show that adipocyte YAP and TAZ are activated during obesity and induce upregulation of anti-apoptotic and downregulation of pro-apoptotic factors such as BIM (Bcl-2 interacting mediator of cell death), and thereby promote adipocyte survival in diet-induced obesity.

\section{Results}

Obesity promotes activation of YAP and TAZ in human and mouse white adipocytes. To test whether YAP and TAZ activity in white adipocytes is affected during obesity, we examined the subcellular localization of YAP and TAZ, as well as YAP/TAZ target gene expression in white adipocytes of humans and mice. In human visceral adipocytes from lean subjects (body mass index $<25$ ), YAP and TAZ were localized equally in the cytosol and nucleus, whereas YAP/TAZ were predominantly detected in the nuclei of human visceral adipocytes from obese subjects (body mass index $>30$ ) (Fig. 1a, b). Quantitative reverse transcriptase PCR (RT-PCR) showed that expression of YAP/TAZ target genes, such as Cyr61, AmotL2, and Lats2, was upregulated in adipocytes of obese subjects (Fig. 1c). Similarly, in adipose tissue from mice fed a standard diet (SD), YAP and TAZ were localized both in the cytosol and nucleus (Fig. 1d, e), whereas 4 weeks of high-fat diet (HFD) feeding resulted in a strong increase in nuclear localization of YAP/TAZ (Fig. 1d, e), both in the epididymal (visceral) and the inguinal (subcutaneous) white adipose tissue (WAT). Quantitative RT-PCR showed increased expression of YAP/TAZ target genes in adipocytes from HFD-fed mice (Fig. 1f). These data indicate that obesity in humans and mice goes along with increased activation of YAP and TAZ in white adipocytes.

Adipocyte-specific YAP and TAZ deficiency leads to increased adipocyte death during obesity. To examine the functions of YAP and TAZ in white adipocytes, we generated inducible adipocyte-specific YAP- and TAZ-deficient mice by crossing mice carrying floxed alleles of Yap and $\mathrm{Taz}\left(\mathrm{Yap}^{\mathrm{fl} / \mathrm{fl}} ; \mathrm{Taz}^{\mathrm{fl} / \mathrm{fl}}\right)$ with the Adipoq-CreER ${ }^{\mathrm{T} 2}$ mouse line ${ }^{41}$. Adipocyte-specific, tamoxifen-

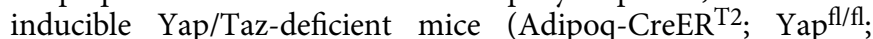
$\mathrm{Taz}^{\mathrm{fl} / \mathrm{fl}}$, hereafter termed iAd-Yap/Taz-KO) showed strong recombination of both floxed alleles in white adipocytes, resulting in loss of YAP and TAZ proteins (Supplementary Fig. 1a). On SD, iAd-Yap/Taz-KO mice showed no difference in total body weight, glucose tolerance, or fat mass compared to wild-type littermates (Supplementary Fig. 1b-d). To test whether activation of YAP and TAZ during obesity affects adipocyte function, iAd-Yap/TazKO mice were fed a HFD. After 12 weeks of HFD feeding, iAdYap/Taz-KO mice gained significantly less weight compared to wild-type littermates (Fig. 2a). Fasting blood glucose levels were similar between both groups, but iAd-Yap/Taz-KO mice showed an improved glucose tolerance compared to wild-type littermates (Fig. 2b). The mass of the visceral WAT (vWAT) and subcutaneous WAT (sWAT) of iAd-Yap/Taz-KO mice was markedly reduced compared to wild-type littermates (Fig. 2c), whereas body size and mass of other tissues including interscapular brown adipose tissue (BAT) was unchanged (Supplementary Fig. 2a, b). These changes were not observed in mice with induced adipocyte-specific loss of YAP or TAZ alone (Fig. 2a, c and Supplementary Fig. 2c), indicating that both transcriptional regulators have overlapping functions in adipocytes. Adipocyte morphology and average cell size were not changed in iAd-Yap/ Taz-KO mice (Fig. 2d and Supplementary Fig. 2d, e), but the total number of adipocytes was strongly reduced in the visceral and sWAT (Fig. 2e). We also found an increased number of crownlike structures and of macrophages as well as an increased number of cells undergoing apoptosis as determined by TUNEL (terminal deoxynucleotidyl transferase dUTP nick end labeling) assay in the WAT of iAd-Yap/Taz-KO mice (Fig. 2d, f-h). These data indicate that loss of YAP and TAZ results in increased adipocyte death during obesity.

Despite very high recombination efficiency in adipocytes resulting in loss of YAP and TAZ shortly after tamoxifen treatment (Supplementary Fig. 1a), we noticed that, after 16 weeks of HFD, Yap/Taz expression was similar to wild-type mice (Supplementary Fig. 2f). To test whether the increased adipocyte cell death due to loss of YAP and TAZ leads to increased adipogenesis resulting in accumulation of newly formed wild-type adipocyte in iAd-Yap/Taz-KO mice, we performed adipocyte pulse-chase experiments ${ }^{42}$. We therefore crossed adipocytespecific, tamoxifen-inducible Cre transgenic mice (Adipoq$\mathrm{CreER}^{\mathrm{T} 2}$ ) with the $\mathrm{mT} / \mathrm{mG}$ Cre reporter line, which switches from membrane-targeted Tomato expression to membranetargeted enhanced green fluorescent protein (EGFP) expression upon Cre-mediated recombination (Fig. 2i) ${ }^{43}$. One week after tamoxifen injection, nearly all adipocytes showed green fluorescence (Supplementary Fig. $2 \mathrm{~g}$ ), and 8 weeks after induction of Cre activity and feeding with SD, $4-5 \%$ of the adipocytes in the vWAT of both wild-type and iAd-Yap/Taz-KO mice were Tomato-positive (Supplementary Fig. 2h). However, whereas in wild-type mice fed an HFD for 8 and 16 weeks, $13 \%$ and $25 \%$ of adipocytes were Tomato-positive (Fig. 2j, k), 26\% and $74 \%$ of adipocytes showed red fluorescence in adipocyte-specific Yap/Taz knockouts (Fig. 2j, k). This indicates that they had formed from Tomato-positive adipocyte precursor cells after induction with 
a
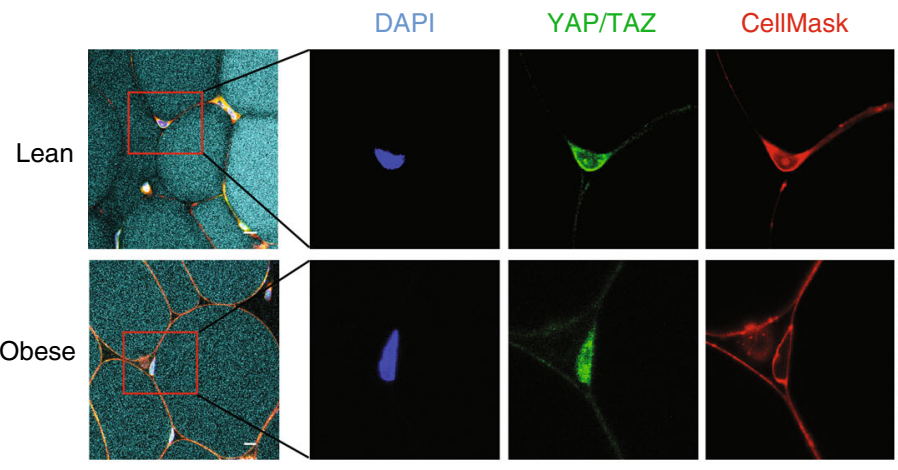

Merge lipid
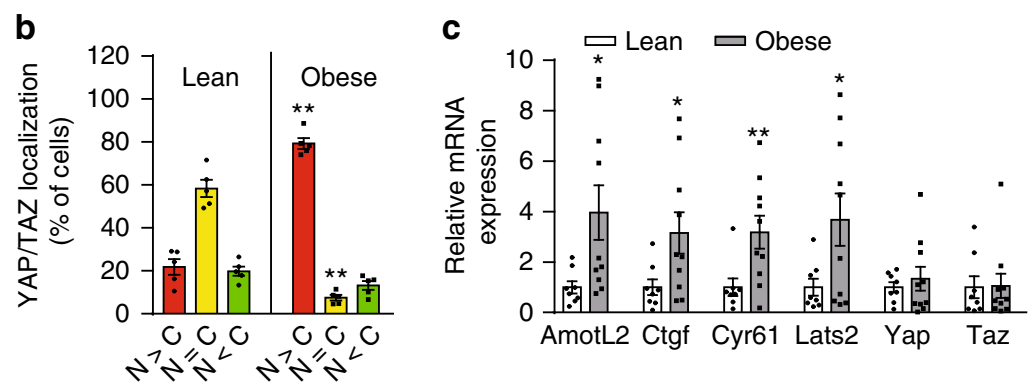

d
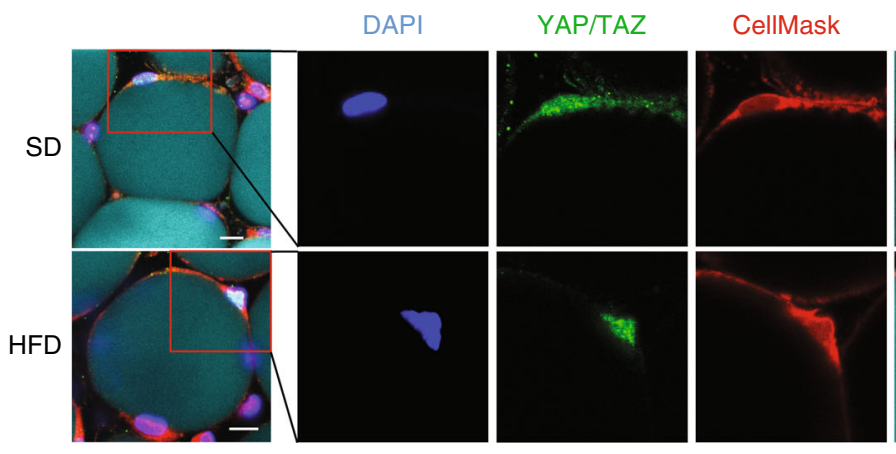

Merge lipid
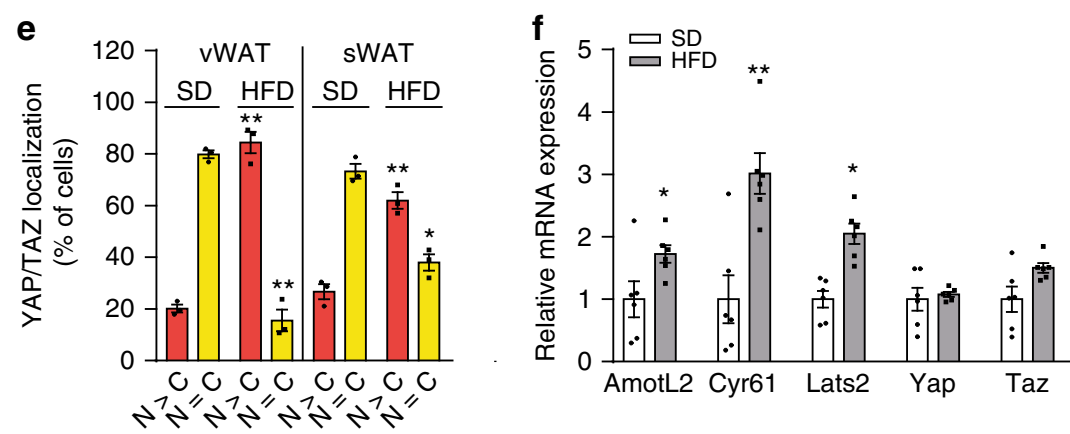

Fig. 1 Obesity promotes YAP/TAZ activation in white adipocytes. a, $\mathbf{d}$ vWAT from lean (body mass index $<25$ ) and obese subjects (body mass index $>$ 30) (a) or from mice fed HFD for 4 weeks and mice fed standard diet (SD) (d) was stained with anti-YAP/TAZ antibody, DAPI, CellMask to visualize adipocyte plasma membranes and LipidTOX (lipid). Scale bars: $10 \mu \mathrm{m}$. b, e Quantification of YAP/TAZ localization to the nucleus (N) or cytosol (C) in visceral white adipocyte of human lean $(n=5)$, and obese subjects $(n=5)(\mathbf{b})$ or in the visceral (vWAT) and subcutaneous white adipose tissue (sWAT) from standard diet (SD)-fed $(n=3)$ and HFD-fed mice $(n=3)(\mathbf{e})$. c, $\mathbf{f}$ Expression of YAP/TAZ target genes, and of Yap and Taz in white adipocyte isolated from human visceral adipocytes of lean $(n=8)$ and obese subjects $(n=10)$ (c) or from the vWAT of mice fed HFD $(n=6)$ or standard diet (SD) $(n=6)$ for 4 weeks $(\mathbf{f})$. Data are presented as the mean \pm SEM; ${ }^{*} p \leq 0.05$ and ${ }^{* *} p \leq 0.01$ (compared to lean humans or standard diet (SD)-fed mice in $\mathbf{b}, \mathbf{e}$ ) (unpaired Student's $t$-test). Source data are provided as a Source Data file.

tamoxifen and HFD feeding. Thus, loss of adipocyte YAP/TAZ in obese iAd-Yap/Taz-KO mice leads to increased adipocyte death and increased adipogenesis resulting in the replacement of the majority of YAP/TAZ-deficient adipocytes by wild-type adipocytes after a few months.
Constitutive loss of adipocyte YAP and TAZ leads to adipocyte death and lipodystrophy under HFD. To avoid replacement of Yap/Taz-deficient adipocytes by wild-type adipocytes in iAd-Yap/ Taz-KO mice, we generated constitutive adipocyte-specific Yap/ Taz-deficient mice by crossing $\mathrm{Yap}^{\mathrm{f} / \mathrm{fl}} ; \mathrm{Taz}^{\mathrm{f} / \mathrm{f}}$ mice with Adipoq-Cre 

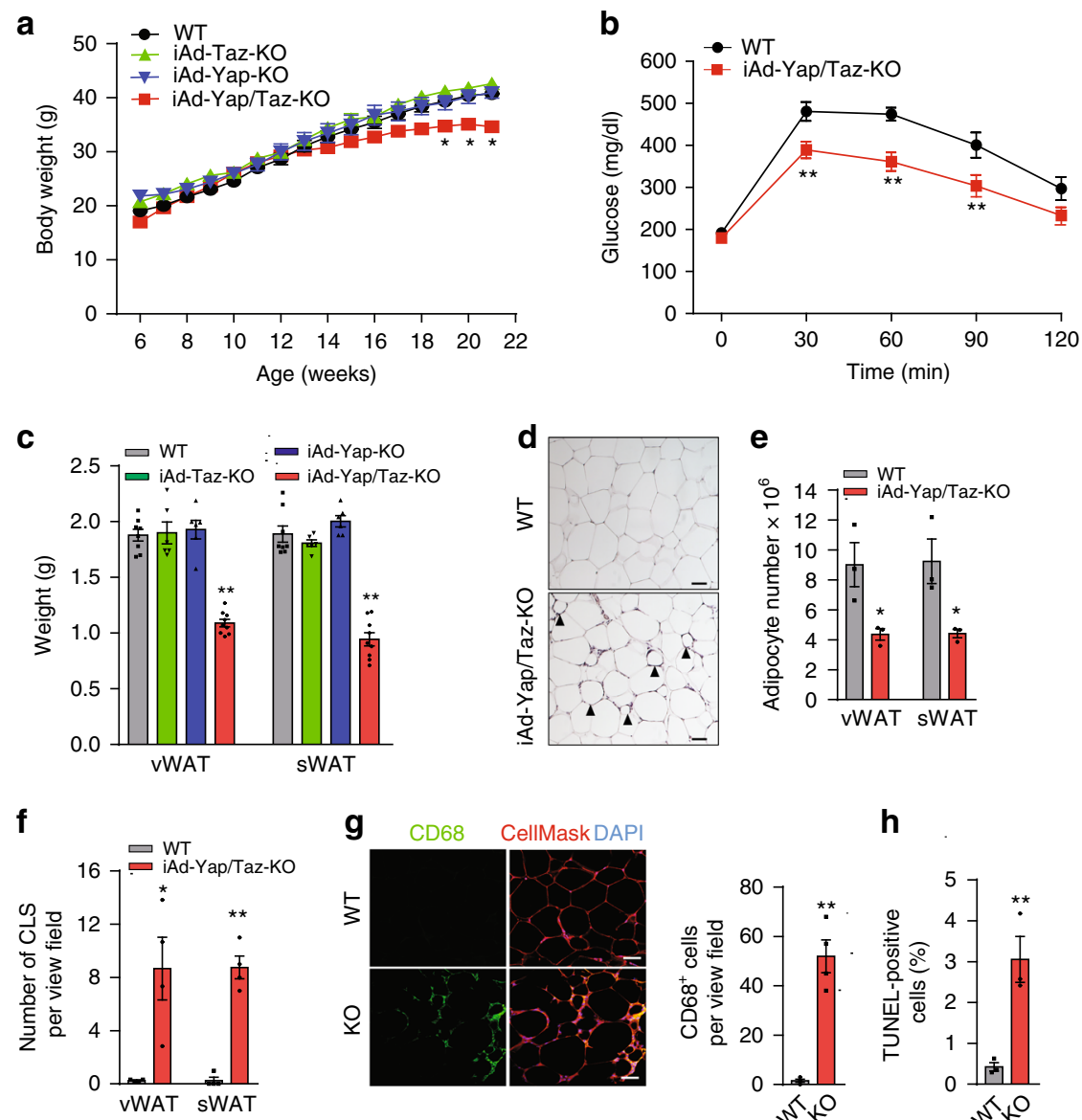

h
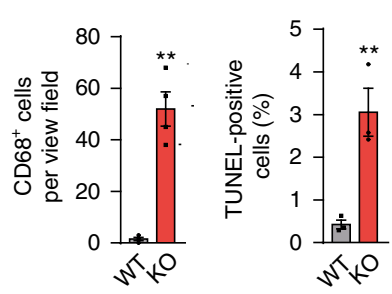

i
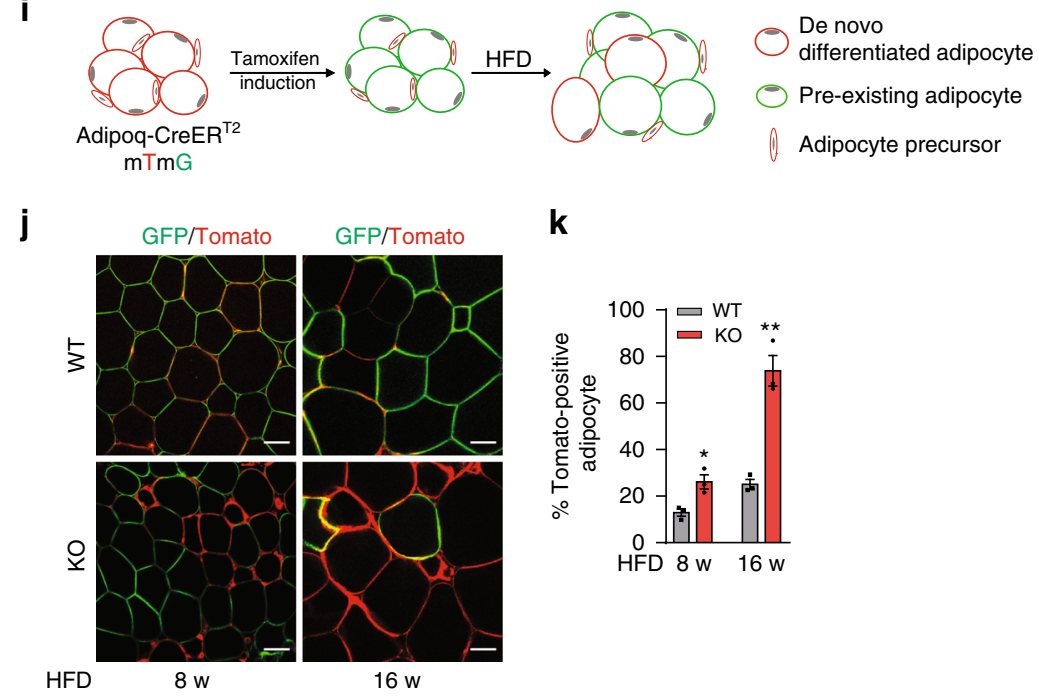

$\mathbf{k}$

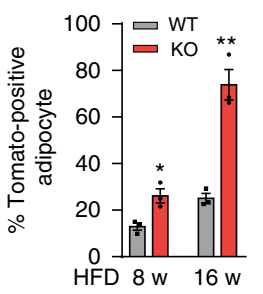

mice $^{44}$ (Adipoq-Cre;Yap ${ }^{\mathrm{fl} / \mathrm{fl}} ; \mathrm{Taz}^{\mathrm{fl} / \mathrm{fl}}$, henceforth termed Ad-Yap/ Taz-KO), which were born at the expected Mendelian ratio without any apparent abnormality. On SD, Ad-Yap/Taz-KO mice showed normal total body weight and glucose tolerance (Supplementary Fig. 3a, b). There was also no difference in fat tissue mass between Ad-Yap/Taz-KO mice and wild-type animals, but Ad-Yap/Taz-KO mice showed a slight increase in crown-like structures in their adipose tissue (Supplementary Fig. 3c, d). However, after 10 weeks of HFD feeding, the total body weight of Ad-Yap/Taz-KO mice started to be significantly lower than that of wild-type littermates (Fig. 3a). This was accompanied by an increased glucose tolerance and a massive reduction in the mass of both vWAT and sWAT in Ad-Yap/Taz-KO mice (Fig. 3b, c). Histological analysis of adipose tissue from HFD-fed Ad-Yap/ Taz-KO mice showed increased numbers of TUNEL-positive adipocytes undergoing apoptosis as well as increased numbers of crown-like structures already after 4 weeks of HFD feeding (Fig. 3d, e). After 14 weeks of HFD feeding, Ad-Yap/Taz-KO mice showed a strongly reduced expression of the adipocytespecific lipid droplet protein perilipin-1, accumulation of macrophages, and increased expression of M2 macrophage marker genes including $A r g 1, M r c 1$, and $I L-10$, whereas expression of M1 marker genes such as iNOS was rather decreased (Fig. $3 \mathrm{f}-\mathrm{h}$ ). Furthermore, expression of several adipocyte marker genes, such 
Fig. 2 Induction of Yap/Taz loss in mature white adipocyte leads to adipose death and increased adipogenesis during HFD feeding. a-c Wild-type (WT, $n=8-9)$, iAd-Yap/Taz-KO ( $n=9-11)$, iAd-Yap-KO $(n=6-7)$, and iAd-Taz-KO $(n=6)$ mice were fed HFD for 16 weeks. Shown are the body weight development (a), glucose tolerance (b), and weight of VWAT and sWAT (c) after 16 weeks of HFD. d H\&E-stained epididymal vWAT sections from WT and iAd-Yap/Taz-KO mice after 16 weeks of HFD. Arrowheads point to crown-like structures. Scale bar: $50 \mu$ m. e Total adipocyte number in vWAT and sWAT from WT and iAd-Yap/Taz-KO mice fed HFD for 16 weeks ( $n=3$ mice in both groups; at least 10 sections were analyzed per animal). $\mathbf{f}$ Number of crown-like structures (CLS) in vWAT and sWAT from WT and iAd-Yap/Taz-KO mice fed HFD for 16 weeks $(n=4$ mice per group; at least 10 view fields were analyzed per animal). $\mathbf{g}$ Sections of vWAT from WT and iAd-Yap/Taz-KO mice stained with anti-CD68 antibody (green), CellMask (red), and DAPI. Scale bar: $50 \mu \mathrm{m}$. The bar diagram shows the statistical evaluation of CD68-positive cells per view field $(n=4$ mice per group; at least 10 sections per animal were analyzed). $\mathbf{h}$ TUNEL-positive cells in sections of vWAT from WT and iAd-Yap/Taz-KO mice $(n=3$ mice per group; at least 10 sections per animal were analyzed). $\mathbf{i}$ Experimental design of analysis of adipogenesis in vivo using Adipoq-CreER ${ }^{\top 2} ; \mathrm{mT} / \mathrm{mG}$ mice. $\mathbf{j}$, $\mathbf{k}$ Representative images (j) and

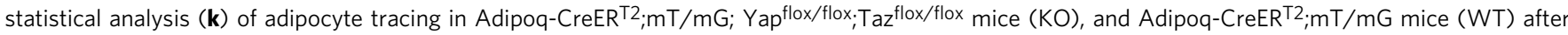
tamoxifen treatment and 8 and 16 weeks of HFD ( $n=3$ mice; at least 10 sections per animal were analyzed). Scale bars: $50 \mu \mathrm{m}$. Shown are mean values \pm SEM; ${ }^{*} p \leq 0.05$ and ${ }^{* *} p \leq 0.01$ (two-way ANOVA in $\mathbf{a}, \mathbf{b}$ and one-way ANOVA in $\mathbf{c}$, and unpaired Student's $t$-test in $\mathbf{e}-\mathbf{h}, \mathbf{k}$ ). Source data are provided as a Source Data file.

as adiponectin (Adipoq), Leptin, Resistin, Glut4, Ppary, and Fabp4, were strongly reduced in the WAT of Ad-Yap/Taz-KO mice (Fig. 3i). Collectively, these data confirm that YAP and TAZ are essential for adipocyte survival during obesity, and that loss of YAP and TAZ leads to adipocyte apoptosis and lipodystrophy.

YAP and TAZ regulate expression of genes encoding pro- and anti-apoptotic factors in adipocytes. The massive increase in apoptotic adipocyte death in obese mice after loss of YAP/TAZ in adipocytes suggests that YAP/TAZ activation during obesity promotes anti-apoptotic, pro-survival pathways, or inhibits proapoptotic pathways. To determine whether YAP/TAZ are involved in regulation of the pro-survival nuclear factor- $\kappa \mathrm{B}$ (NF$\kappa \mathrm{B})$ signaling pathway, we analyzed the phosphorylation of NF$\kappa B$ in adipose tissue lysates from Ad-Yap/Taz-KO mice fed HFD for 4 weeks but found no obvious difference in phosphorylation of P65 compared to controls (Supplementary Fig. 4a). Deficiency of YAP/TAZ also did not alter tumor necrosis factor- $\alpha$ (TNFa)induced P65 phosphorylation in primary adipocytes from wildtype and Ad-Yap/Taz-KO mice fed HFD for 4 weeks (Supplementary Fig. $4 \mathrm{~b}$ ). This indicates that YAP/TAZ are not critically involved in NF- $\mathrm{KB}$ activation during obesity.

To understand the underlying mechanism by which YAP/TAZ modulate adipocyte survival during obesity, we performed expression profiling by RNA sequencing (RNA-seq) and quantitative RT-PCR using total RNA isolated from white adipocytes of Ad-Yap/Taz-KO mice and of wild-type littermates, which had been fed HFD for 4 weeks. As expected, YAP/TAZ target genes, such as Ctgf, Cyr61, AmotL2, and Lats2, showed reduced expression in Ad-Yap/Taz-KO adipocytes (Fig. 4a and Supplementary Fig. 4c). In addition, we found that the antiapoptotic genes $B c l 2$ and $B c l 2 l 2$ were downregulated, whereas the pro-apoptotic gene Bim was upregulated in the absence of Yap/ Taz (Fig. 4a and Supplementary Fig. 4c). Interestingly, in adipocytes from HFD-fed wild-type animals as well as from the vWAT of obese humans, the pro-apoptotic factor BIM (BCL2L11) was downregulated compared to lean controls (Fig. 4b, c), and in chromatin immunoprecipitation (ChIP) assays we found that YAP showed increased binding to a TEAD-binding motif in the promoter region of Bim in adipocytes from HFD-fed mice compared to animals fed an SD (Fig. 4d). The strong upregulation of Bim expression in the absence of YAP/TAZ was confirmed on the protein level in primary adipocytes isolated from HFD-fed Ad-Yap/Taz-KO mice, as well as in adipocytes differentiated from the SVF (stromal vascular fraction) of AdYap/Taz-KO mice in vitro (Fig. 4b, e, f), which also showed increased apoptosis (Fig. 4g). Suppression of Yap and Taz expression in differentiated 3T3-L1 adipocytes also resulted in increased Bim expression and apoptosis (Fig. $4 \mathrm{~h}-\mathrm{j}$ ), and small interfering RNA (siRNA)-mediated suppression of Bim expression blocked the increase in apoptosis (Fig. 4i, j). Consistent with this, suppression of Bim expression in epididymal adipocytes of HFD-fed Ad-Yap/Taz-KO mice in vivo by viral transduction of a corresponding short hairpin RNA (shRNA) resulted in a significantly reduced number of crown-like structures and of TUNEL-positive cells (Fig. 4k-m), and was accompanied by a significant decrease in fat loss (Supplementary Fig. 4d). These data indicate that YAP/TAZ suppress expression of the proapoptotic gene Bim to promote adipocyte survival.

TNFa and IL-1 $\beta$ induce YAP/TAZ nuclear translocation and YAP/TAZ transcriptional activity. To identify signals in fat tissue that might induce YAP/TAZ activation during obesity, we analyzed whether palmitic acid or several cytokines, including TNFa, interleukin (IL)-1 $\beta$, CCL2, and IL-6 are able to induce YAP/TAZ target gene expression in 3T3-L1 adipocytes. Quantitative RT-PCR analysis showed that expression of the YAP/TAZ target genes Ctgf, Cyr61, and Ankrd1 was induced by TNFa or IL$1 \beta$ (Fig. 5a). Consistent with this, we detected increased YAP/ TAZ nuclear translocation in response to TNFa and IL-1 $\beta$ (Fig. 5b and Supplementary Fig. 5a), and suppression of Yap/Taz expression strongly inhibited TNFa- and IL-1 $\beta$-induced YAP/ TAZ target gene expression (Fig. $5 \mathrm{c}$ and Supplementary Fig. $5 \mathrm{~b}$ ). An involvement of TNF $\alpha$ and IL-1 $\beta$ in the activation of YAP and TAZ in mouse adipocytes during obesity could be demonstrated by treating animals with the TNFa inhibitor etanercept and the IL-1 $\beta$ inhibitor anakinra. In HFD-fed mice, etanercept and anakinra significantly reduced HFD-induced nuclear translocation of $\mathrm{YAP} / \mathrm{TAZ}$, as well as upregulation of YAP/TAZ target gene expression (Fig. 5d, e). In differentiated adipocytes, TNFa or IL$1 \beta$ did not change protein or phosphorylation levels of YAP/TAZ (Supplementary Fig. 5c) and knockdown of the Hippo pathway kinase LATS1/2 did not affect induction of YAP/TAZ target gene expression by TNFa or IL-1 $\beta$ (Supplementary Fig. 5d). This indicates that regulation of YAP/TAZ activity by TNF $\alpha$ or IL-1 $\beta$ is independent of the Hippo pathway. Inhibition of RhoA, which was activated by TNFa in 3T3-L1 adipocytes (Supplementary Fig. 5e) by C3-exoenzyme, depolymerization of actin by latrunculin B, and inhibition of myosin II ATPase by blebbistatin attenuated TNF $\alpha$ - and IL-1 $\beta$-induced nuclear translocation of YAP/TAZ (Fig. $5 \mathrm{f}$ and Supplementary Fig. 5f-h), as well as TNFa- and IL$1 \beta$-induced YAP/TAZ target gene expression (Fig. $5 \mathrm{~g}$, $\mathrm{f}$ and Supplementary Fig. 5i, j). To examine which RhoGEF protein is involved in TNFa-induced RhoA activation in adipocytes, we focused on ArhGEF2 (GEF-H1), which has been described to be activated through the TNFa receptor ${ }^{45}$, on ArhGEF11 (PDZRhoGEF), which has been involved in adipocyte metabolism $^{46}$, as well as on the related RhoGEF proteins ArhGEF1 and 
a

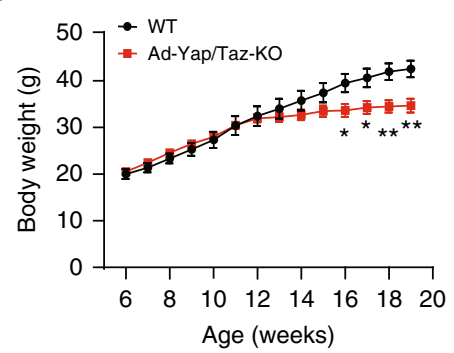

b

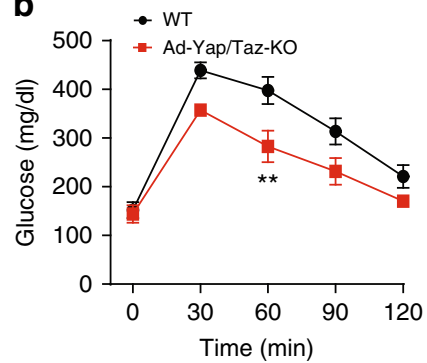

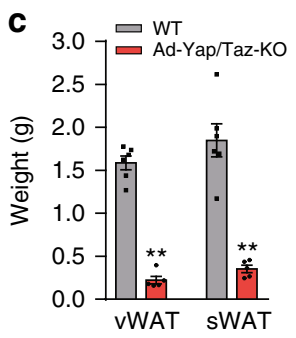
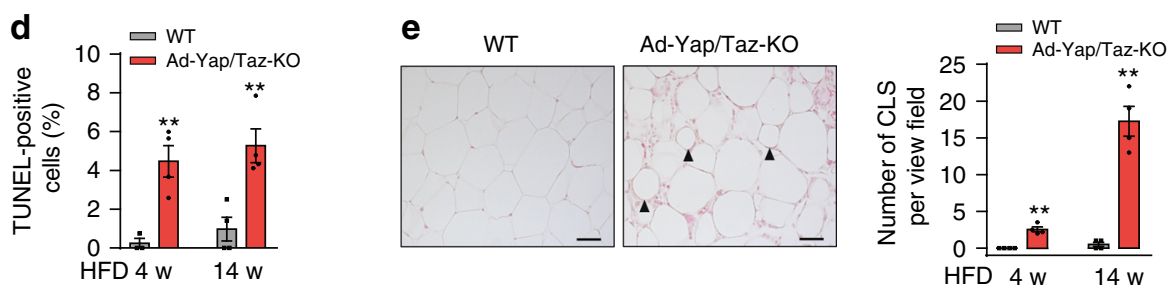

$\mathbf{f}$
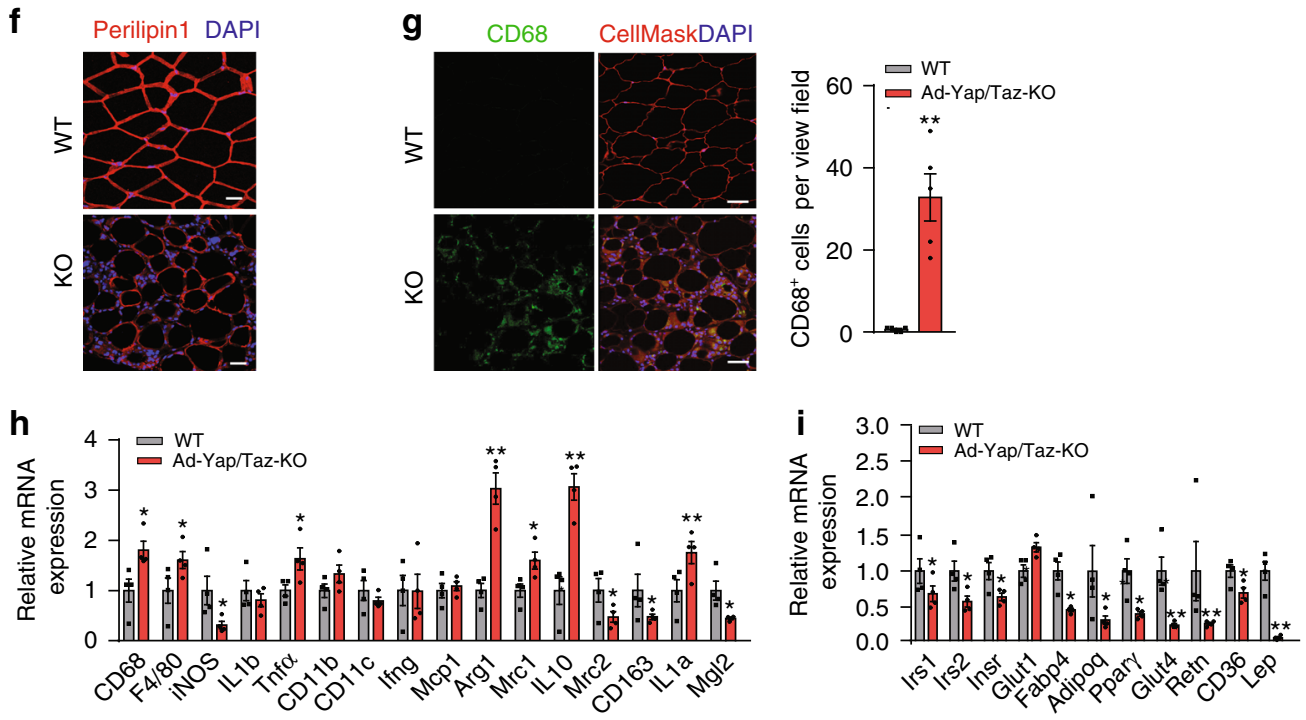

Fig. 3 Ad-Yap/Taz-KO mice exhibit increased adipocyte cell death and lipodystrophy. a-c Wild-type (WT, $n=6)$ and Ad-Yap/Taz-KO mice $(n=5-8)$ were fed HFD for 14 weeks starting at 6 weeks of age. Shown are the development of body weight (a), glucose tolerance after 12 weeks of HFD (b), as well as weight of vWAT and SWAT (c). d, e Wild-type (WT, $n=3-4)$ and Ad-Yap/Taz-KO mice $(n=4)$ were fed HFD for 4 or 14 weeks (w). Shown are the quantification of TUNEL-positive cells in sections of vWAT from WT and Ad-Yap/Taz-KO mice (d), and H\&E-stained epididymal vWAT sections (14 weeks) (e). Arrowheads in e indicate crown-like structures (CLS). Scale bar: $50 \mu \mathrm{m}$. The bar diagram (e) shows the number of CLS in vWAT from WT and Ad-Yap/Taz-KO mice ( $n=4$ mice in both groups; at least 10 sections were analyzed per animal). $\mathbf{f}, \mathbf{g}$ Paraffin sections of vWAT from WT and AdYap/Taz-KO mice stained with anti-Perilipin-1 (red) and DAPI (f) or with anti-CD68 antibody (green), CellMask (red), and DAPI (g). Scale bars: $50 \mu$ m. The bar diagram shows the statistical evaluation of $\mathrm{CD} 68^{+}$cells per view field ( $n=5$ mice per group; at least 10 sections were analyzed per animal). $\mathbf{h}$, i Quantitative RT-PCR showing mRNA expression of M1 and M2 macrophage marker genes (h) and of adipocyte-specific marker genes (i) in vWAT from WT and Ad-Yap/Taz-KO mice fed HFD for 14 weeks ( $n=4$ mice per group). Data are presented as the mean \pm SEM; ${ }^{\star} p \leq 0.05$ and ${ }^{\star *} p \leq 0.01$ (twoway ANOVA in $\mathbf{a}, \mathbf{b}$ and unpaired Student's $t$-test in $\mathbf{c}-\mathbf{e}, \mathbf{g}$-i). Source data are provided as a Source Data file.

ArhGEF12 (LARG). Whereas knockdown of ArhGEF1, ArhGEF11, and ArhGEF12 had no effect on TNFa-induced upregulation of YAP/TAZ target genes (Supplementary Fig. 5k), knockdown of ArhGEF2 blocked the TNFa effect (Fig. 5i). This suggests that ArhGEF2- and RhoA-mediated actomyosin contractility is required for TNF $\alpha$ - and IL-1 $\beta$-induced YAP/TAZ nuclear translocation and subsequent transcriptional activity.

To identify additional intracellular signaling pathways activated by TNFa and IL-1 $\beta$, which are involved in YAP/TAZ activation by both cytokines in adipocytes, we tested the effect of various protein kinase inhibitors on TNF $\alpha$ - and IL- $1 \beta$-induced YAP/TAZ target gene expression. Whereas inhibition of mitogenactivated protein-kinase kinase, inhibitor of NF-kB-kinase, phosphoinositide-3-kinase, and p38 kinase had no effect (Supplementary Fig. 6a), inhibition of c-Jun $\mathrm{N}$-terminal kinase (JNK) strongly attenuated TNFa- and IL-1 $\beta$-induced YAP/TAZ target gene expression (Fig. 6a and Supplementary Fig. 6b). A requirement of JNK for TNF $\alpha$ - and IL-1 $\beta$-induced YAP/TAZ transcriptional activity was validated by knockdown of Jnk, which also blocked TNF $\alpha$ - and IL-1 $\beta$-induced activation of YAP/TAZ target genes (Fig. 6b and Supplementary Fig. 6c). C3-exoenzyme and latrunculin B did not affect TNFa-induced JNK phosphorylation (Supplementary Fig. 6d), indicating that Rho-mediated signaling is not required for TNFa- and IL- $1 \beta$-induced JNK activation. Although disruption of the actin cytoskeleton by latrunculin B abolished TNF $\alpha$ - and IL- $1 \beta$-induced YAP/TAZ 
a

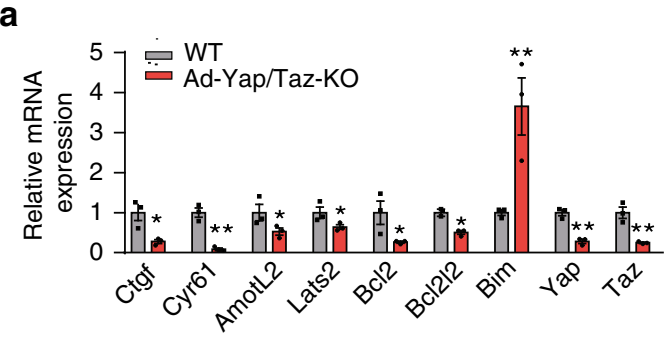

b

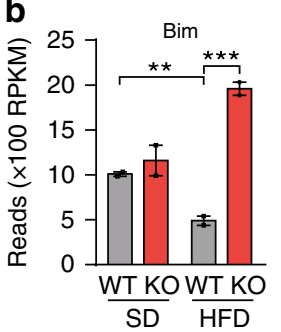

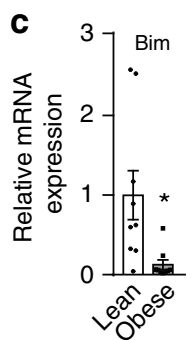

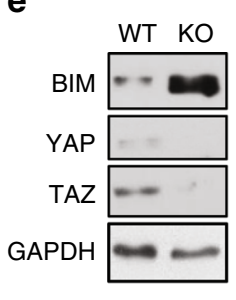

$\mathbf{f}$

$\mathbf{g}$

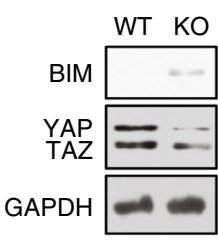

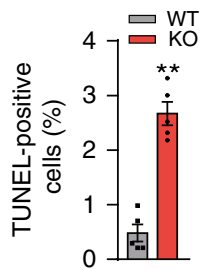

h

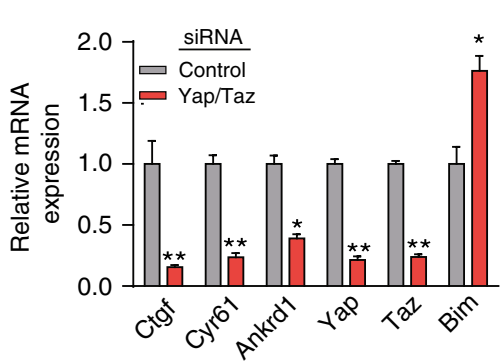

i

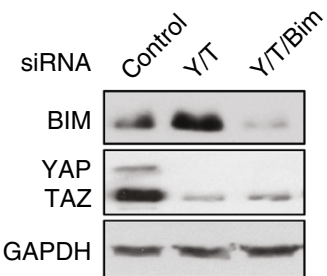

j

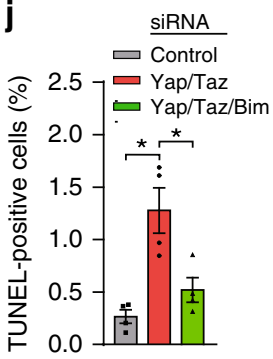

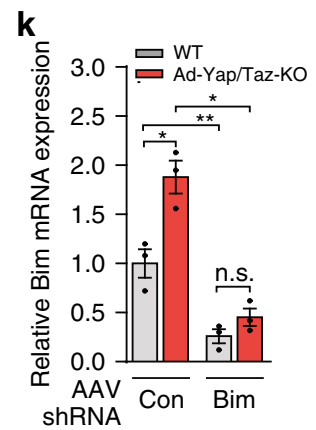

I
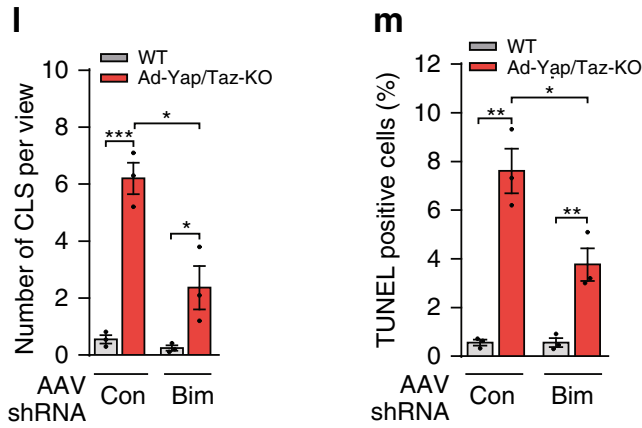

Fig. 4 YAP/TAZ promote adipocyte survival by suppression of Bim expression. a Quantitative RT-PCR showing mRNA expression of Yap, Taz, and their target genes including pro- and anti-apoptotic factors in white adipocytes isolated from WT and Ad-Yap/Taz-KO mice fed HFD for 4 weeks $(n=3$ mice per group). b RNA-seq data showing expression of Bim (Bcl2/11) in white adipocytes isolated from WT and Ad-Yap/Taz-KO mice fed HFD or standard diet for 4 weeks ( $n=2$ mice per group). c Expression of Bim in isolated adipocytes from the vWAT of lean $(B M I<25)$ and obese humans $(B M I>30)(n=9$ in both groups). d Chromatin immunoprecipitation (ChIP) PCR of the TEAD-binding site in the Bim promoter region in lysates of vWAT prepared from WT mice fed standard diet (SD) or HFD for 4 weeks using an anti-YAP antibody (\#4912, Cell Signaling Technology) ( $n=4$ mice per group). e, $\mathbf{f}$ Western blot analysis showing BIM protein level in white adipocyte isolated from WT and Ad-Yap/Taz-KO mice (KO) fed HFD for 4 weeks (e) and in adipocytes differentiated from the SVF of WT and Ad-Yap/Taz-KO mice (KO) (f). $\mathbf{g}$ Apoptotic cell death detected by TUNEL staining of adipocytes differentiated from SVF of WT and Ad-Yap/Taz-KO mice ( $n=5$ mice per group). $\mathbf{h}$ Quantitative RT-PCR showing mRNA expression of YAP/TAZ target genes in differentiated 3T3-L1 adipocytes treated with control siRNA or siRNA directed against Yap and Taz $(n=3)$. i, j Differentiated 3T3-L1 adipocytes were treated with control siRNA or siRNA directed against Yap, Taz, and Bim, and western blot analysis was performed to analyze protein levels of BIM, YAP, TAZ, and GAPDH (i). In parallel, cells were analyzed by TUNEL staining to determine cell apoptosis $(\mathbf{j})(n=4)$. $\mathbf{k}$ Bim expression in adipocytes isolated from vWAT 4 weeks after injection of adeno-associated virus transducing control shRNA (AAV-Con) or shRNA directed against Bim (AAV-shBim) fed HFD ( $n=3$ mice per group). I, $\mathbf{m}$ Quantification of crown-like structures (CLS) (I) and of TUNEL-positive cells ( $\mathbf{m}$ ) in sections of vWAT from WT and AdYap/Taz-KO mice 4 weeks after injection of adeno-associated virus transducing control shRNA (AAV-Con) or shRNA directed against Bim (AAV-shBim) and fed HFD ( $n=3$ mice per group, at least 10 sections were analyzed per animal). Data are presented as the mean \pm SEM; ${ }^{*} p \leq 0.05$ and ${ }^{\star *} p \leq 0.01$ (unpaired Student's t-test in $\mathbf{a}, \mathbf{c}, \mathbf{g}, \mathbf{h}$ and one-way ANOVA in $\mathbf{b}, \mathbf{d}, \mathbf{j}-\mathbf{m}$ ). Source data are provided as a Source Data file. 
a

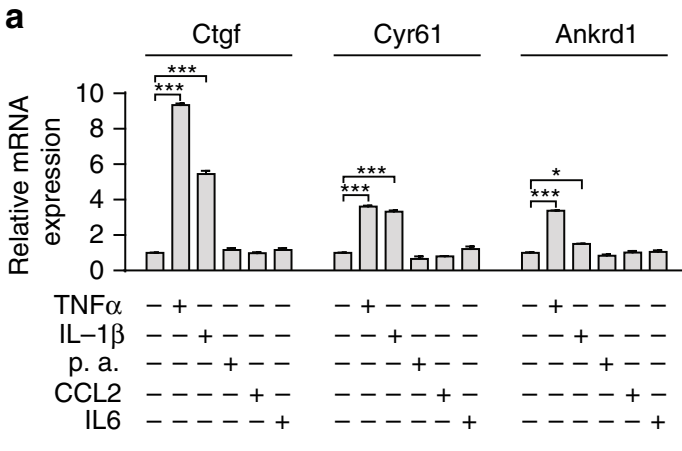

d

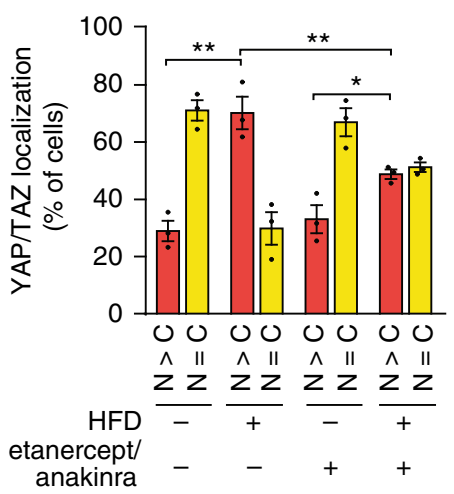

e

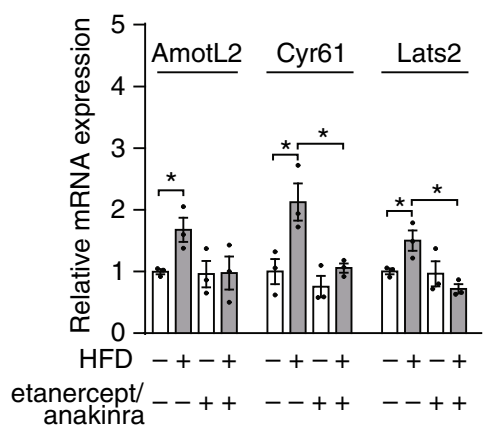

g

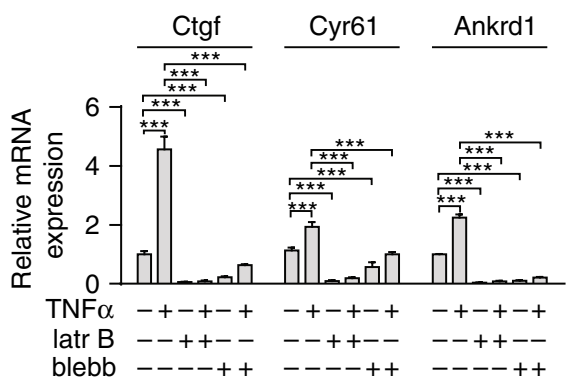

b

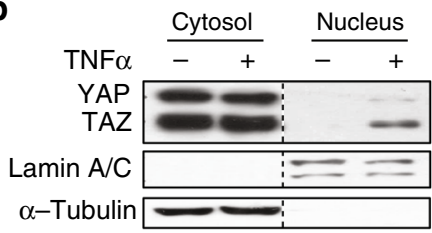

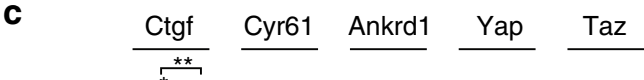

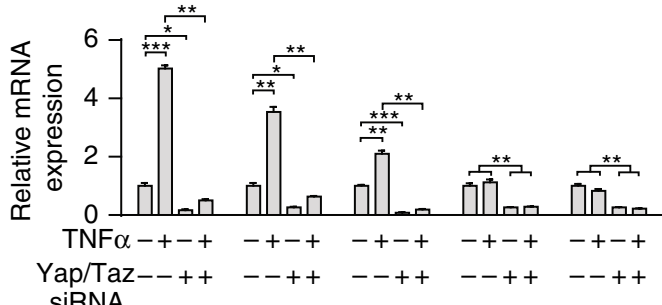
siRNA

f
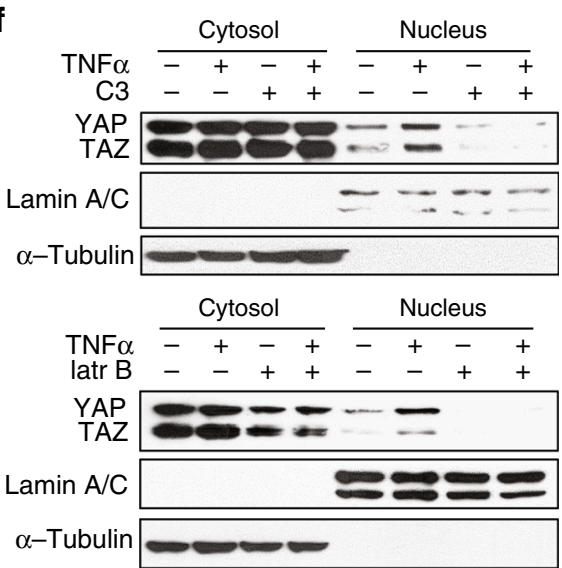

h
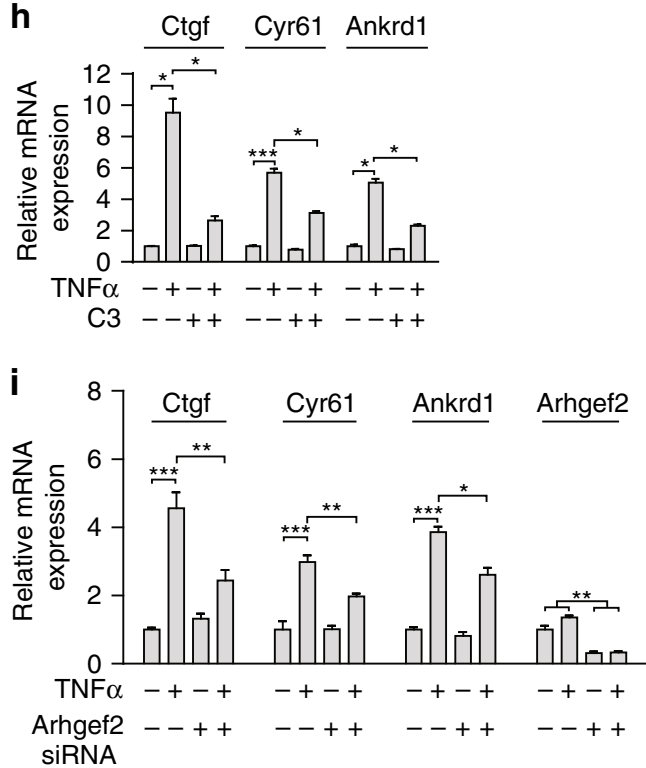

siRNA-mediated suppression of c-Jun affects TNF $\alpha$ - and IL- $1 \beta$ induced YAP/TAZ target gene expression. We found that suppression of c-Jun expression attenuated TNFa- and IL- $1 \beta$ induced YAP/TAZ target gene expression (Fig. $6 \mathrm{~d}$ and Supplementary Fig. 6e), which indicates that JNK and c-JUN mediate TNFa-induced YAP/TAZ transcriptional activity. Taken together, these data suggest that RhoA-mediated actomyosin contractility nuclear localization, inhibition of JNK activation had no effect (Figs. $5 \mathrm{f}$ and $6 \mathrm{c}$, and Supplementary Fig. 5g), indicating that JNK does not regulate YAP/TAZ function on the level of nuclear translocation. As the YAP/TAZ/TEAD complex has been shown to functionally interact with AP-1 that synergistically regulates YAP/TAZ target gene expression ${ }^{47}$, and as JNK phosphorylates and regulates C-Jun, a component of AP-1 ${ }^{48}$, we tested whether 
Fig. 5 TNF $\alpha$ regulates YAP/TAZ transcriptional activity in adipocytes. a Quantitative RT-PCR showing mRNA expression of YAP/TAZ target genes in response to TNF $\alpha(1.1 \mathrm{nM}), \mathrm{IL}-1 \beta(5.9 \mathrm{nM}), \mathrm{IL}-6(0.8 \mathrm{nM}), \mathrm{CCL} 2(1.2 \mathrm{nM})$, and palmitic acid (p.a., $500 \mathrm{nM})$ in differentiated 3T3-L1 adipocyte $(n=3$ per group). b Differentiated 3T3-L1 adipocytes were incubated without or with $1.1 \mathrm{nM} \mathrm{TNF} \alpha$ for $6 \mathrm{~h}$, then cytosolic and nuclear fractions were analyzed by immunoblotting using antibodies against YAP/TAZ, $\alpha$-Tubulin (cytoplasmic marker), and Lamin A/C (nuclear marker). The dashed line indicates the margins of a region, which was cut out. $\mathbf{c}, \mathbf{g}, \mathbf{h}$ Differentiated 3T3-L1 adipocytes were treated with control siRNA or siRNA directed against Yap/Taz (c) or were pretreated with $2.5 \mu \mathrm{M}$ latrunculin B (latr B), $20 \mu \mathrm{M}$ blebbistatin (blebb) for $30 \mathrm{~min}(\mathbf{g})$, or with $1 \mu \mathrm{g} / \mathrm{ml}$ of C3-exoenzyme (h) for $6 \mathrm{~h}$. Thereafter, cells were incubated without or with $1.1 \mathrm{nM}$ TNF $\alpha$ for $8 \mathrm{~h}$ and expression of YAP/TAZ target genes was analyzed $(n=3)$. d Quantification of YAP/TAZ localization to the nucleus (N) or cytosol (C) in the visceral (vWAT) from standard diet (SD)-fed $(n=3)$ and HFD-fed mice $(n=3)$ treated without or with etanercept and anakinra by intraperitoneal injection. e Expression of YAP/TAZ target genes in white adipocyte isolated from the vWAT of mice fed HFD $(n=3)$ or standard diet (SD) and treated without or with etanercept and anakinra $(n=3)$. f Differentiated 3T3-L1 adipocytes were preincubated with C3exoenzyme (C3) or latrunculin B (latr B). Thereafter, cells were incubated without or with $1.1 \mathrm{nM}$ TNF $\alpha$ for $6 \mathrm{~h}$, and cytosolic and nuclear fractions were analyzed by immunoblotting using antibodies against YAP, TAZ, Lamin A/C, and $\alpha$-Tubulin. i Differentiated 3T3-L1 adipocytes were treated with control siRNA or siRNA directed against Arhgef2. Thereafter, cells were incubated in the absence or presence of TNF $\alpha$ ( $1.1 \mathrm{nM})$ and expression of YAP/TAZ target genes was determined $(n=4)$. Data are presented as the mean \pm SEM; ${ }^{\star} p \leq 0.05$, ${ }^{\star \star} p \leq 0.01$, and ${ }^{\star \star \star} p \leq 0.001$ (one-way ANOVA). Source data are provided as a Source Data file.

a

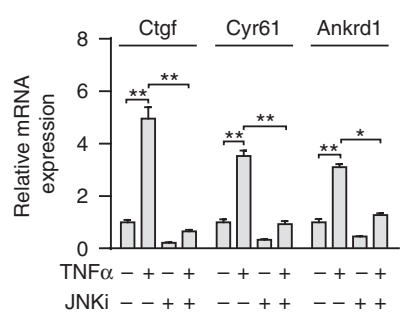

b

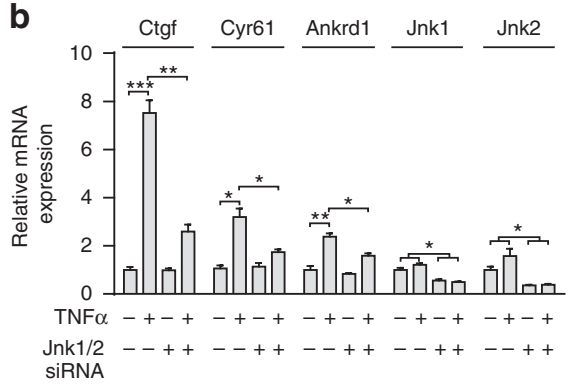

C

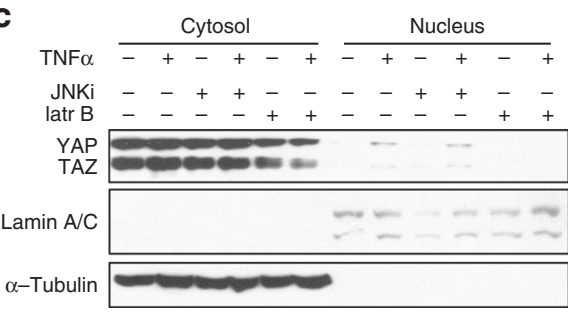

d

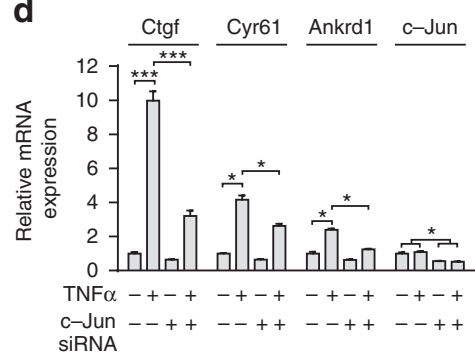

Fig. 6 JNK and c-JUN mediate TNF $\boldsymbol{\alpha}$-induced YAP/TAZ transcriptional activity. a-d Differentiated 3T3-L1 adipocytes were incubated in the absence or presence of the JNK inhibitor SP600125 (JNKi, $5 \mu \mathrm{M})(\mathbf{a}, \mathbf{c})$, latrunculin B) (latr B, $2.5 \mu \mathrm{M})(\mathbf{c})$, or were treated with control siRNA or siRNA directed against Jnk1 and 2 (b) or c-Jun (d). Thereafter, cells were incubated in the absence or presence of TNF $\alpha(1.1 \mathrm{nM})$, and expression of YAP/TAZ target genes was determined $(n=3)(\mathbf{a}, \mathbf{b}, \mathbf{d})$ or cytosolic and nuclear fractions were analyzed by immunoblotting using antibodies against YAP and TAZ, Lamin A/C or $\alpha$ Tubulin (c). Shown are mean values mean $\pm \mathrm{SEM} ;{ }^{\star} p \leq 0.05,{ }^{\star \star} p \leq 0.01$, and ${ }^{\star \star *} p \leq 0.001$ (one-way ANOVA). Source data are provided as a Source Data file.

is required for TNF $\alpha$ - and IL-1 $\beta$-induced YAP/TAZ nuclear localization, whereas JNK/c-JUN is essential for YAP/TAZ transcriptional activity.

\section{Discussion}

YAP and TAZ are transcriptional regulators that integrate metabolic pathways, mechanical cues, and various signaling pathways to control different cellular functions including proliferation, differentiation, and cell survival ${ }^{27-29}$. Based on our initial observation that YAP and TAZ, as well as a characteristic YAP/TAZ transcriptional program were activated in mouse and human white adipocytes during the development of obesity, we found that loss of YAP and TAZ expression did not affect adipocyte function in normal healthy mice. However, in animals fed a HFD, loss of YAP and TAZ resulted in lipodystrophy including massive adipocyte death, a compensatory increase in adipogenesis and infiltration by immune cells. This indicates that YAP/TAZ signaling protects mature adipocytes from cell death during obesity.

Given the YAP/TAZ activation in adipocytes during obesity, the question arises how YAP/TAZ are activated during expansion of the adipose tissue. As adipocytes increase in size during obesity, they experience increased mechanical stress due to compressive forces or strain exerted by the extracellular matrix on expanding adipocytes ${ }^{49,50}$. There is also an increase in connective fiber content in obese adipose tissue, which results in an increase in overall rigidity of the adipose tissue and which has been shown to contribute to increased cell death in the obese adipose tissue ${ }^{51}$. In fact, YAP and TAZ activity is controlled by mechanical signals, such as extracellular matrix rigidity, shear stress, and stretching $35,52,53$, and cytoskeletal tension has been linked to YAP/TAZ activity $28,35-37$. A stiff environment favors YAP nuclear localization and activation, whereas attachment to soft substrates increases cytoplasmic retention of YAP ${ }^{35}$. Recent work has shown that integrin adhesion to the extracellular matrix and 
integrin signaling, as well as RhoA-mediated signaling and actomyosin-driven contractility play important roles in mechanical regulation of YAP/TAZ activity ${ }^{28,54-58}$, which appears to be largely independent of the Hippo pathway ${ }^{35,59}$. In addition, actomyosin-mediated tension has been shown to regulate the thermogenic capacity of brown adipocytes through YAP and $\mathrm{TAZ}^{60}$. However, it is not known whether a similar mechanism exists in white adipocytes. Our data show that inhibition of RhoA-mediated signaling, latrunculin B-induced depolymerization of actin, and myosin II ATPase inhibition by blebbistatin reduced cytokine-induced YAP/TAZ activation in differentiated adipocytes, suggesting that increased cytoskeletal tension in obese adipocytes contributes to YAP/TAZ activation during obesity.

The enlargement of the adipose tissue during obesity is accompanied by the development of a chronic low-grade inflammation of the adipose tissue, which includes infiltration of the adipose tissue by macrophages and increased levels of cytokines $^{13-19}$. By testing several mediators that are increased in the obese adipose tissue, we identified TNF- $\alpha$ and IL-1 $\beta$, which have been shown to be produced by adipocytes and cells of the SVF including infiltrating macrophages in obese mice and humans ${ }^{61-65}$, as potent activators of adipocyte YAP/TAZ. This suggests that inflammatory mediators produced in the obese adipose tissue contribute to adipocyte YAP/TAZ activation during obesity. TNF- $\alpha$ and IL- $1 \beta$ can activate several intracellular signaling pathways including extracellular signal-regulated kinase, IKK/NF- $\mathrm{kB}$-signaling, $\mathrm{p} 38$, and $\mathrm{JNK}^{66}$. By pharmacological inhibition and siRNA-mediated knockdown, we identified JNK as a critical signaling component in cytokine-induced YAP/TAZ activation in adipocytes. Although inhibition of JNK had no effect on cytokine-induced nuclear translocation of YAP/TAZ, it strongly inhibited cytokine-induced activation of the YAP/TAZ transcriptional program, which we found to also require c-JUN, a component of AP- $1^{48}$. As the YAP/TAZ/TEAD complex has been shown to functionally interact with AP-1 to synergistically regulate target gene expression ${ }^{47}$, we conclude that a similar mechanism is also operating in adipocytes. As a major mechanism mediating cytokine-induced YAP/TAZ nuclear translocation, we identified RhoA-mediated actomyosin contractility, whereas blockade of the Hippo pathway component LATS1/2 had no effect on cytokine-induced YAP/TAZ nuclear translocation. This is consistent with several reports indicating that Rho/actomyosinmediated nuclear translocation of YAP/TAZ occurs independently of the Hippo pathway ${ }^{34,35,67 .}$

Expansion of the adipose tissue during obesity is accompanied by increased mechanical, metabolic, and inflammatory stress, as well as increased adipocyte cell death ${ }^{68}$. As we observed massive apoptotic adipocyte cell death in the absence of YAP/TAZ in mature adipocytes during obesity, activation of YAP/TAZ during obesity may either promote anti-apoptotic, pro-survival pathways or inhibit pro-apoptotic pathways. The inflammatory NF- $\mathrm{B}$ pathway is also important for adipocyte survival during obesity, as loss of signaling components of this pathway results in increased apoptotic death of adipocytes during obesity 24,26 . We could largely exclude that YAP/TAZ have an effect on IKK/NF$\kappa \mathrm{B}$ pro-survival signaling, as loss of YAP/TAZ did not affect TNF- $\alpha$-induced activation of NF- $\kappa B$ in vitro and as NF- $\kappa B$ activity was not altered in adipocytes from obese Ad-Yap/Taz-KO mice compared to controls.

When analyzing transcriptomic changes in white adipocytes of Ad-Yap/Taz-KO mice fed HFD for 4 weeks compared to wildtype littermates, we observed that the anti-apoptotic genes $\mathrm{Bcl} 2$ and $B c l 2 l 2$ were downregulated, and that the pro-apoptotic gene Bim was strongly upregulated in the absence of YAP/TAZ. This is consistent with previous data, which showed that, during embryonic stem cell (ESC) differentiation, YAP attenuates apoptosis primarily through transcriptional upregulation of antiapoptotic factors, including BCL2, BCL2L1, and MCL-1 ${ }^{69}$, as well as with observations, which showed that YAP/TAZ promote cell survival through suppression of the pro-apoptotic protein BIM, whereas YAP/TAZ knockdown increased BIM expression ${ }^{70}$. Similarly, it has been shown that overexpression of YAP or TAZ suppresses BIM expression ${ }^{71,72}$. In differentiated adipocytes in vitro, we could show that increased apoptotic cell death induced by knockdown of YAP/TAZ, which was accompanied by increased BIM expression, could be rescued by knockdown of Bim. Thus, we conclude that increased activation of YAP/TAZ in obese adipocytes suppresses expression of pro-apoptotic BIM and promotes expression of anti-apoptotic factors such as $\mathrm{Bcl} 2$ and $\mathrm{Bcl} 2 \mathrm{12}$, and that these transcriptional regulatory effects contribute to the protective effect of YAP/TAZ against increased adipocyte cell death during obesity. Targeting YAP/TAZ signaling may provide a novel therapeutic strategy to treat obesity by slowing the expansion of the white adipose mass during hypercaloric diet.

\section{Methods}

Reagents. Tamoxifen (catalog number T5648), collagenase II (catalog number C6885), wortmannin (catalog number W3144), latrunculin B (catalog number 428020), and IL-6 (catalog number I1395) were from Sigma-Aldrich. SC514 was from Santa Cruz Biotechnology (catalog number 354812-17-2). SP600125 (catalog number S5567) and p38 MAPK inhibitor III (catalog number 506148) were obtained from Merck Chemicals GmbH. Exoenzyme C3 transferase was obtained from Cytoskeleton (catalog number CT03-A). Mouse TNFa was purchased from PeproTech (catalog number 315-01 A). IL-1 $\beta$ was from Life Technologies GmbH (catalog number 10139-HNAE). CCL2 (catalog number 479-JE-10) was from BioTechne $\mathrm{GmbH}$. The following antibodies were obtained from Cell Signaling Technology: anti-YAP/TAZ (\#8418, 1:1000), anti-YAP (\#4912, 1:1000), antiphospho-YAP (Ser127) (\#4911, 1:1000), anti-phospho-TAZ (Ser89) (\#59971, 1:1000), anti-Lamin A/C (\#2032, 1:1000), anti-GAPDH (\#2118, 1:3000), antiphospho-JNK (Thr183/Tyr185) (\#9251, 1:1000), anti-IкBa (\#4812, 1:1000), antiP65 (\#4764, 1:1000), anti-phospho-P65 (Ser536) (\#3033, 1:1000), and anti-BIM (\#2933, 1:1000). Anti-TAZ (\#HPA007415, 1:1000) and anti- $\alpha$-Tubulin (\#T9026, 1:3000) were from Sigma-Aldrich. Anti-mouse and rabbit horseradish peroxidase (HRP)-conjugated secondary antibodies were from Cell Signaling Technology.

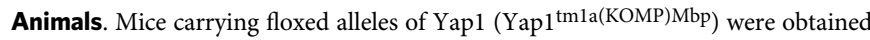
from the Knockout Mouse Project (KOMP) Repository (KOMP, Davis, California, USA). ESCs carrying a targeted allele of Taz (Wwtr $1^{\text {tmla }}$ ), which after removal of a cassette flanked by FRT (flippase recognition target) sites allows for Cre-mediated recombination, were purchased from EUCOMM (Helmholtz Zentrum München). ESC clones were injected into C57BL/6 blastocysts and were transferred to pseudopregnant females. Chimeric offspring were bred with C57BL/6 mice to produce heterozygous animals. Germline transmission was confirmed in the $\mathrm{F}_{1}$ generation using a PCR genotyping strategy. To delete the selection cassette, mice were crossed to Flp-deleter mice ${ }^{73}$. Adipocyte-specific Yap/Taz-deficient mice (iAd-Yap/TazKO) were generated by crossing Yap ${ }^{\text {flox/flox;Taz }}{ }^{\text {flox/flox }}$ mice with Adipoq-CreER ${ }^{\mathrm{T} 2}$ transgenic mice ${ }^{41}$. For adipocyte pulse-chase experiments, iAd-Yap/Taz-KO and control animals were crossed with the Cre reporter line Gt(ROSA)

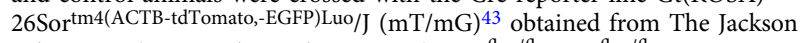
Laboratory (JAX stock number 007676). Yap ${ }^{\text {flox/flox;Taz }}$ flox/flox mice were also mated with Adipoq-Cre mice ${ }^{44}$ to generate Ad-Yap/Taz-KO mice. For induction of Cre-mediated recombination in iAd-Yap/Taz-KO mice, $1 \mathrm{mg}$ of tamoxifen was injected intraperitoneally for 5 consecutive days at the age of 5 weeks. Animals were fed SD (SC, Altromin) or a Western-type HFD containing $30 \%$ fat (ssniff) starting from the age of 6 weeks with water ad libitum. Tamoxifen-treated Yap ${ }^{\text {flox }}$ flox; Taz flox/flox littermates served as controls. For in vivo experiments, male animals were used at the age of 8-12 weeks if not stated otherwise, whereas adipose tissue from male mice were tested in several in vitro experiments as indicated. Animal care and experimental procedures followed were compliant with ethical regulations and were approved by the local animal welfare authorities and committees (Regierungspräsidia Karlsruhe and Darmstadt).

Cell culture. Primary white adipocytes and stromal vascular cell fraction from mouse epididymal fat pads and human primary white adipocytes were isolated as described previously ${ }^{24}$. All human samples were collected with the informed consents from the donors following the bioethics and safety regulations. Isolated primary white adipocytes were cultured at $37^{\circ} \mathrm{C}$ and $5 \% \mathrm{CO}_{2}$ in Dulbecco's modified Eagle's medium with $10 \%$ fetal bovine serum and antibiotic solution. 3T3-L1 preadipocytes (obtained from American Type Culture Collection) were differentiated into mature adipocytes as previously described ${ }^{74}$. 
siRNA-mediated knockdown. A suspension of differentiated 3T3-L1 adipocytes was transfected with siRNA using Lipofectamine RNAiMax transfection reagent (Invitrogen) according to the manufacturer's instruction and seeded on collagencoated plates. The targeted sequences of mouse siRNAs directed against RNAs encoding Yap, Taz, Lats1, Lats2, Jnk1, Jnk2, c-Jun, Bim, Rhogef1, Rhogef2, Rhogef11, and Rhogef12 were as follows: Yap, 5'-GCTAGATAAAGAAAGCTTT-3' and 5'-CCAATAGTTCCGATCCCTT-3'; Taz, 5'-GAATCAGCCTCTGAATCAT$3^{\prime}$ and $5^{\prime}$-GTCTGAAGATCTGATCCCT-3'; Lats1, 5'-GCAACATTCAATT AACCGA-3'; Lats2, 5'-CCATGAAGACTCTCAGGAA-3'; Jnk1, 5'-CAAGA GATTTGTTATCCAA-3', 5'-GAAGCAAACGTGACAACAA-3', 5'-GTTCTTAT GAAGTGTGTTA- $3^{\prime}$, and 5'-TAAATACGCTGGATATAGC-3'; Jnk2, 5'-CC GCAGAGTTCATGAAGAA-3', 5'-GCGGATCTCTGTGGACGAA-3', 5'-AAA GAGAGCATGCGATTGA- $3^{\prime}$, and 5'-GCATTCAGCTGGTATCATT-3'; c-Jun, 5'-AGCGTTCGTTATCACAATAAA-3', 5'-GTGCCTACGGCTACAGTAA-3', and $5^{\prime}$-GTAACCCTAAGATCCTAAA-3'; Bim, $5^{\prime}$-GTGTTTACATGTGG CGTGT- $3^{\prime}$ and $5^{\prime}$-GCCAATTTATGGATGATTT- ${ }^{\prime}$; Arhgefl, $5^{\prime}$-TTGGCT

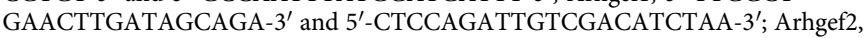
$5^{\prime}$-CACCAAGGCCTTAAAGCTCTA- $3^{\prime}$ and $5^{\prime}$-ATCAATCTTTATGGACT TCTA-3'; Arhgef11, 5'-CAGAAGTTTATCAGCAGACAA-3' and 5'-CTGGGA GATTCTACACCTGAA-3'; Arhgef12, 5'-CAGCAGCTTGTTAGCAGAGAA-3' and $5^{\prime}$-AAGAATTCAAATATTAAATAA-3'.

Glucose tolerance test. Intraperitoneal glucose tolerance test and insulin toler ance test were performed as described previously ${ }^{24}$. Briefly, after overnight fasting, glucose was administered intraperitoneally at a dose of $1.5 \mathrm{mg}$ glucose per g body weight for HFD-fed mice and $2 \mathrm{mg}$ glucose per g body weight for mice fed SD. Blood glucose levels were measured in blood taken from the tail vein prior to or at the indicated times after glucose administration using a glucometer (Accu-Chek Aviva, Roche). Insulin sensitivity was determined by intraperitoneal injection of 0.75-1 units of insulin per kg body weight and measuring of glucose levels in blood taken from the tail vein before and at the indicated times after insulin injection in mice fasted for $6 \mathrm{~h}$.

Adipocyte pulse-chase experiments. Adipocyte pulse-chase experiments were performed as described ${ }^{42}$. Mice were treated with $1 \mathrm{mg} / \mathrm{d}$ tamoxifen at the age of 5 weeks by intraperitoneal injection for five consecutive days. Mice were then placed on HFD or remained on SD for an 8-week or a 16-week chase period. Inguinal and epididymal WAT were taken from several regions throughout the depot and were analyzed by whole mount confocal microscopy for tdTomato and eGFP expression. Per mouse, at least 300 (sWAT) or 2000 (vWAT) adipocytes were counted from multiple images.

Administration of adeno-associated virus. Adeno-associated viral (AAV) vectors of serotype 8, carrying a scrambled sequence (AAV-Ctrl) or an shRNA directed against the mouse Bim RNA (AAV-Bim-shRNA) were obtained from Vigen Bioscience. For delivery of virus to the epididymal WAT, mice were anesthetized with $2 \%$ isoflurane and a laparotomy was performed to expose the epididymal fat pad under a sterile hood. Virus were diluted to $2.5 \times 10^{10}$ viral genomes $/ \mathrm{ml}$ in sterile phosphate-buffered saline (PBS) and a total amount of $50 \mu \mathrm{l} \mathrm{Bim} \mathrm{shRNA}$ virus or control virus was injected at five different sites of each fat pad using a $0.3 \mathrm{~cm}^{3} 31 \mathrm{G}$ insulin syringe.

Etanercept and anakira treatment in vivo. Mice fed HFD for 4 weeks were injected during the fourth week with $10 \mathrm{mg} / \mathrm{kg}$ etanercept and $25 \mathrm{mg} / \mathrm{kg}$ anakira intraperitoneally for 7 consecutive days. Afterwards, animals were killed for fat tissue collection and adipocyte isolation, or whole mount tissue staining.

Histology and immunohistochemistry. Tissues were fixed by paraformaldehyde (4\%), dehydrated in an ethanol gradient, and then transferred to xylene solution for embedding in paraffin. Five-micrometer sections were stained with hematoxylineosin and images were analyzed with light microscopy. Adipocyte area in sections was determined in more than 100 cells per condition using ImageJ (NIH). Adipocyte number was calculated as described previously ${ }^{75}$. TUNEL assay was performed on paraffin sections $(5 \mu \mathrm{m})$ of WAT following the instructions of the In Situ Cell Death Detection Kit (Roche Applied Sciences). For immunohistochemical staining, paraffin sections $(5 \mu \mathrm{m})$ were placed in a microwave oven for heatinduced epitope retrieval. The following antibodies were used: anti-CD68 (Bio-rad, MCA1957, 1:100) and anti-Perilipin-1 (Cell Signaling Technology, 3470, 1:100). CellMask $^{\text {Th }}$ Orange plasma membrane stain (Life Technologies, Thermo Fisher Scientific, C10045) was used to visualize the plasma membrane. Whole fat tissue mount for YAP/TAZ localization (using anti-YAP/TAZ antibody \#8418, 1:100, Cell Signaling Technology) and adipocyte pulse-chase experiments was performed as described $^{76}$. Immunofluorescence imaging was performed using a Leica TCS SP5 confocal microscope.

Quantitative RT-PCR and RNA-seq. Total RNA was isolated from adipocytes or fat tissue using the RNeasy lipid tissue Mini Kit (Qiagen, Valencia, CA, USA) and DNA was removed using the QIAGEN RNase-Free DNase Set following the manufacturer's instructions. cDNA was synthesized using ProtoScript ${ }^{\circledR}$ II reverse transcriptase (New England Biolabs). Quantitative RT-PCR was performed using LightCycler 480 Real-Time PCR detection System (Roche Molecular Biochemicals, Mannheim, Germany) according to the manufacturer's instructions. Relative mRNA expression was normalized to GAPDH. PCR primers are listed under Supplementary Table 1. For RNA-seq, RNA and library preparation integrity were verified with a BioAnalyzer 2100 (Agilent) or LabChip Gx Touch 24 (Perkin Elmer). Twenty nanograms of total RNA was used as starting material for ribosomal depletion with RiboGone-Mammalian (Clontech) followed by library preparation using SMARTer Stranded Total RNA Sample Prep Kit (Clontech). Sequencing was performed on the NextSeq500 instrument (Illumina) using v2 chemistry, resulting in an average of $35 \mathrm{M}$ reads per library with $1 \times 75 \mathrm{bp}$ single end setup. The resulting raw reads were assessed for quality, adapter content, and duplication rates with FastQC (Andrews S. 2010, FastQC: a quality control tool for high-throughput sequence data. Available online at: http://www.bioinformatics babraham.ac.uk/projects/fastqc). Reaper version 15-065 was employed to trim reads after a quality drop below a mean of Q20 in a window of 20 nucleotides ${ }^{77}$. Only reads between 15 and 75 nucleotides were cleared for further analyses. Trimmed and filtered reads were aligned vs. the Ensembl mouse genome version mm10 (GRCm38) using STAR 2.5.2b with the parameter

"-outFilterMismatchNoverLmax 0.1 " to increase the maximum ratio of mismatches to mapped length to $10 \%^{78}$. The number of reads aligning to genes was counted with featureCounts 1.5.1 tool from the Subread package $\mathrm{e}^{79}$. Only reads mapping at least partially inside exons were admitted and aggregated per gene. Reads overlapping multiple genes or aligning to multiple regions were excluded. Differentially expressed genes were identified using DESeq2 version $1.14 .1^{80}$. Only genes with a minimum fold change of $\pm 1.5(\log 2 \pm 0.59)$, a maximum Benjamini-Hochberg corrected $p$-value of 0.05 , and a minimum combined mean of 5 reads were deemed to be significantly differentially expressed. The Ensemble annotation was enriched with UniProt data (release 24.03.2017) based on Ensembl gene identifiers $^{81}$

Chromatin immunoprecipitation. ChIP analysis was performed as described previously ${ }^{82}$. vWAT from mice fed with SD or HFD for 4 weeks was washed with PBS and crosslinked with $1 \%$ formaldehyde for $10 \mathrm{~min}$. Samples were lysated in adipocyte lysis buffer (5 mM PIPES pH 8.0, $85 \mathrm{mM} \mathrm{KCl}$, and $0.5 \% \mathrm{NP}-40$ ) and homogenized using a Retsch MM 300 TissueLyser Mixer Mill. Then, samples were centrifuged ( $5 \mathrm{~min}, 700 \times \mathrm{g}, 4^{\circ} \mathrm{C}$ ), pelleted nuclei were resuspended in SDS lysis buffer (1\% SDS, $10 \mathrm{mM}$ EDTA $\mathrm{pH} 8.1$, and $50 \mathrm{mM}$ Tris. $\mathrm{HCl} \mathrm{pH} \mathrm{8.1)} \mathrm{and} \mathrm{sheared}$ with a Bandelin Sonopuls hd 2070 (18 pulses, each pulse $10 \mathrm{~s}$ at $100 \%$ of the sonicator's amplitude) to generate DNA fragments of 200-1000 bp length. The chromatin was precleared and incubated with YAP antibody (\#4912, Cell Signaling Technology) or IgG overnight under rotation. Complexes were pulled down with $20 \mu \mathrm{l}$ Magnet ChIP A beads (Millipore) and eluted in elution buffer containing 1\% SDS and $100 \mathrm{mM} \mathrm{NaHCO}$. Samples were digested with proteinase K, and DNA was extracted using PCR Clean-up kit (Macherey-Nagel). Samples were analyzed

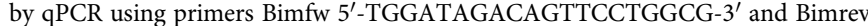
$5^{\prime}$-CGGGCACGACAAGCATATAA- $3^{\prime}$

Western blottings. The nuclear and cytoplasmatic fraction of adipocytes were separated using NE-PER ${ }^{\mathrm{im}}$ Nuclear and Cytoplasmic Extraction Reagents (Thermo Scientific). Mouse fat tissues or adipocytes were collected, snap-frozen in liquid nitrogen and stored at $-80^{\circ} \mathrm{C}$. For protein extraction, $\sim 50 \mathrm{mg}$ frozen tissue was homogenized for $2 \mathrm{~min}$ at $30 \mathrm{~Hz}$ using a Retsch MM 300 TissueLyser Mixer Mill in $200 \mu$ lysis buffer $(50 \mathrm{mM}$ Tris pH 7.5, 2 mM EDTA, 2 mM EGTA, 0.5 M mannitol, $1 \%$ Triton $\mathrm{X}-100$, as well as phosphatase inhibitors $(10 \mathrm{mg} / \mathrm{ml}$ leupeptin, pepstatin A, 4-(2-aminoethyl) benzenesulfonyl-fluoride, and aprotinin) and protease inhibitors (PhosSTOP, Roche) $)^{83}$. Extracts were spun down, and fat layer and cell debris were removed. Proteins were separated by SDS-polyacrylamide gel electrophoresis and analyzed by immunoblotting using the indicated antibodies. For detection, Pierce ${ }^{\text {TM }}$ ECL Western Blotting Substrate (Thermo Scientific) or Immobilon Western Chemiluminescent HRP Substrate (Merck) were used.

Study approval. The work on human adipocyte samples was approved by the ethical committee of Xi' an Jiaotong University (XJTU2018-249 and XJTU2019-12) and conforms to the guidelines of the 2000 Helsinki declaration. Written informed consent was obtained from all subjects before their participation. All procedures of animal care and use in this study were approved by the local animal welfare authorities and committees (Regierungspräsidia Karlsruhe and Darmstadt).

Statistics. Trial experiments or experiments done previously were used to determine sample size with adequate statistical power. Samples were excluded in cases where RNA/cDNA quality or tissue quality after processing was poor (below commonly accepted standards). Animals were excluded from experiments if they showed any signs of sickness. The investigator was blinded to the group allocation and during the experiment. Data represent biological replicates. In all studies, comparison of mean values was conducted with unpaired, two-tailed Student's $t$ test or one-way or two-way analysis of variance with Bonferroni's post hoc test. In all analyses, statistical significance was determined at the $5 \%$ level $(P<0.05)$. 
Depicted are mean values \pm SEM as indicated in the figure legends. Statistical analysis was performed with Prism5 or Prism6 (GraphPad), or Excel (Microscoft) software.

Reporting summary. Further information on research design is available in the Nature Research Reporting Summary linked to this article.

\section{Data availability}

The RNA-seq data are available in GEO repository under the accession GSE138911. Source data are provided with this paper.

Received: 22 November 2019; Accepted: 5 October 2020; Published online: 28 October 2020

\section{References}

1. Qatanani, M. \& Lazar, M. A. Mechanisms of obesity-associated insulin resistance: many choices on the menu. Genes Dev. 21, 1443-1455 (2007).

2. Rosen, E. D. \& Spiegelman, B. M. Adipocytes as regulators of energy balance and glucose homeostasis. Nature 444, 847-853 (2006).

3. Bluher, M. Obesity: global epidemiology and pathogenesis. Nat. Rev. Endocrinol. 15, 288-298 (2019).

4. Gonzalez-Muniesa, P. et al. Obesity. Nat. Rev. Dis. Prim. 3, 17034 (2017)

5. Csige, I. et al. The impact of obesity on the cardiovascular system. J. Diabetes Res. 2018, 3407306 (2018).

6. Bianchini, F., Kaaks, R. \& Vainio, H. Overweight, obesity, and cancer risk. Lancet Oncol. 3, 565-574 (2002).

7. Wang, Q. A., Tao, C., Gupta, R. K. \& Scherer, P. E. Tracking adipogenesis during white adipose tissue development, expansion and regeneration. Nat. Med. 19, 1338-1344 (2013).

8. Crewe, C., An, Y. A. \& Scherer, P. E. The ominous triad of adipose tissue dysfunction: inflammation, fibrosis, and impaired angiogenesis. J. Clin. Invest. 127, 74-82 (2017)

9. Marcelin, G., Silveira, A. L. M., Martins, L. B., Ferreira, A. V. \& Clement, K. Deciphering the cellular interplays underlying obesity-induced adipose tissue fibrosis. J. Clin. Invest. 129, 4032-4040 (2019).

10. Ghaben, A. L. \& Scherer, P. E. Adipogenesis and metabolic health. Nat. Rev. Mol. Cell Biol. 20, 242-258 (2019).

11. Berry, R., Jeffery, E. \& Rodeheffer, M. S. Weighing in on adipocyte precursors. Cell Metab. 19, 8-20 (2014).

12. Cristancho, A. G. \& Lazar, M. A. Forming functional fat: a growing understanding of adipocyte differentiation. Nat. Rev. Mol. Cell Biol. 12 , 722-734 (2011).

13. Gregor, M. F. \& Hotamisligil, G. S. Thematic review series: adipocyte biology. Adipocyte stress: the endoplasmic reticulum and metabolic disease. J. Lipid Res. 48, 1905-1914 (2007).

14. Rudich, A., Kanety, H. \& Bashan, N. Adipose stress-sensing kinases: linking obesity to malfunction. Trends Endocrinol. Metab. 18, 291-299 (2007).

15. Reilly, S. M. \& Saltiel, A. R. Adapting to obesity with adipose tissue inflammation. Nat. Rev. Endocrinol. 13, 633-643 (2017).

16. Rutkowski, J. M., Stern, J. H. \& Scherer, P. E. The cell biology of fat expansion. J. Cell Biol. 208, 501-512 (2015)

17. Trayhurn, P. Hypoxia and adipocyte physiology: implications for adipose tissue dysfunction in obesity. Annu. Rev. Nutr. 34, 207-236 (2014).

18. $\mathrm{Xu}, \mathrm{H}$. et al. Chronic inflammation in fat plays a crucial role in the development of obesity-related insulin resistance. J. Clin. Invest. 112, 1821-1830 (2003).

19. Weisberg, S. P. et al. Obesity is associated with macrophage accumulation in adipose tissue. J. Clin. Invest. 112, 1796-1808 (2003)

20. Eguchi, A. \& Feldstein, A. E. Adipocyte cell death, fatty liver disease and associated metabolic disorders. Dig. Dis. 32, 579-585 (2014).

21. Eguchi, A. \& Feldstein, A. E. Lysosomal cathepsin D contributes to cell death during adipocyte hypertrophy. Adipocyte 2, 170-175 (2013).

22. Cinti, S. et al. Adipocyte death defines macrophage localization and function in adipose tissue of obese mice and humans. J. Lipid Res. 46, 2347-2355 (2005).

23. Alkhouri, N. et al. Adipocyte apoptosis, a link between obesity, insulin resistance, and hepatic steatosis. J. Biol. Chem. 285, 3428-3438 (2010).

24. Sassmann-Schweda, A. et al. Increased apoptosis and browning of TAK1 deficient adipocytes protects against obesity. JCI Insight 1, e81175 (2016).

25. Luk, C. T. et al. FAK signalling controls insulin sensitivity through regulation of adipocyte survival. Nat. Commun. 8, 14360 (2017).

26. Park, S. H. et al. IKKbeta is essential for adipocyte survival and adaptive adipose remodeling in obesity. Diabetes 65, 1616-1629 (2016).
27. Totaro, A., Panciera, T. \& Piccolo, S. YAP/TAZ upstream signals and downstream responses. Nat. Cell Biol. 20, 888-899 (2018).

28. Panciera, T., Azzolin, L., Cordenonsi, M. \& Piccolo, S. Mechanobiology of YAP and TAZ in physiology and disease. Nat. Rev. Mol. Cell Biol. 18, 758-770 (2017).

29. Koo, J. H. \& Guan, K. L. Interplay between YAP/TAZ and metabolism. Cell Metab. 28, 196-206 (2018).

30. Ma, S., Meng, Z., Chen, R. \& Guan, K. L. The Hippo pathway: biology and pathophysiology. Annu. Rev. Biochem. 88, 577-604 (2019).

31. Piccolo, S., Dupont, S. \& Cordenonsi, M. The biology of YAP/TAZ: Hippo signaling and beyond. Physiol. Rev. 94, 1287-1312 (2014)

32. Zheng, Y. \& Pan, D. The Hippo signaling pathway in development and disease. Dev. Cell 50, 264-282 (2019).

33. Yu, F. X. et al. Regulation of the Hippo-YAP pathway by G-protein-coupled receptor signaling. Cell 150, 780-791 (2012).

34. Feng, $X$. et al. Hippo-independent activation of YAP by the GNAQ uveal melanoma oncogene through a trio-regulated rho GTPase signaling circuitry. Cancer Cell 25, 831-845 (2014).

35. Dupont, S. et al. Role of YAP/TAZ in mechanotransduction. Nature 474, 179-183 (2011).

36. Gaspar, P. \& Tapon, N. Sensing the local environment: actin architecture and Hippo signalling. Curr. Opin. Cell Biol. 31, 74-83 (2014).

37. Wada, K., Itoga, K., Okano, T., Yonemura, S. \& Sasaki, H. Hippo pathway regulation by cell morphology and stress fibers. Development 138, 3907-3914 (2011).

38. Zhao, B., Li, L., Lei, Q. Y. \& Guan, K. L. The Hippo-YAP pathway in organ size control and tumorigenesis: an updated version. Genes Dev. 24, 862-874 (2010).

39. Pan, D. J. The Hippo signaling pathway in development and cancer. Dev. Cell 19, 491-505 (2010)

40. Lin, K. C., Park, H. W. \& Guan, K. L. Regulation of the Hippo pathway transcription factor TEAD. Trends Biochem. Sci. 42, 862-872 (2017).

41. Sassmann, A., Offermanns, S. \& Wettschureck, N. Tamoxifen-inducible Cremediated recombination in adipocytes. Genesis 48, 618-625 (2010).

42. Jeffery, E., Church, C. D., Holtrup, B., Colman, L. \& Rodeheffer, M. S. Rapid depot-specific activation of adipocyte precursor cells at the onset of obesity. Nat. Cell Biol. 17, 376-385 (2015).

43. Muzumdar, M. D., Tasic, B., Miyamichi, K., Li, L. \& Luo, L. Q. A global double-fluorescent cre reporter mouse. Genesis 45, 593-605 (2007).

44. Eguchi, J. et al. Transcriptional control of adipose lipid handling by IRF4. Cell Metab. 13, 249-259 (2011)

45. Waheed, F. et al. Central role of the exchange factor GEF-H1 in TNF-alphainduced sequential activation of Rac, ADAM17/TACE, and RhoA in tubular epithelial cells. Mol. Biol. Cell 24, 1068-1082 (2013).

46. Chang, Y. J. et al. The Rho-guanine nucleotide exchange factor PDZ-RhoGEF governs susceptibility to diet-induced obesity and type 2 diabetes. Elife 4, e06011 (2015)

47. Zanconato, F. et al. Genome-wide association between YAP/TAZ/TEAD and AP-1 at enhancers drives oncogenic growth. Nat. Cell Biol. 17, 1218-1227 (2015).

48. Derijard, B. et al. JNK1: a protein kinase stimulated by UV light and Ha-Ras that binds and phosphorylates the c-Jun activation domain. Cell 76, 1025-1037 (1994)

49. Pope, B. D., Warren, C. R., Parker, K. K. \& Cowan, C. A. Microenvironmental control of adipocyte fate and function. Trends Cell Biol. 26, 745-755 (2016).

50. Shoham, N. \& Gefen, A. Mechanotransduction in adipocytes. J. Biomech. 45 1-8 (2012).

51. Khan, T. et al. Metabolic dysregulation and adipose tissue fibrosis: role of collagen VI. Mol. Cell Biol. 29, 1575-1591 (2009)

52. Codelia, V. A., Sun, G. \& Irvine, K. D. Regulation of YAP by mechanical strain through Jnk and Hippo signaling. Curr. Biol. 24, 2012-2017 (2014).

53. Zhong, W. et al. YAP-mediated regulation of the chondrogenic phenotype in response to matrix elasticity. J. Mol. Histol. 44, 587-595 (2013).

54. Elbediwy, A. et al. Integrin signalling regulates YAP and TAZ to control skin homeostasis. Development 143, 1674-1687 (2016).

55. Elosegui-Artola, A. et al. Mechanical regulation of a molecular clutch defines force transmission and transduction in response to matrix rigidity. Nat. Cell Biol. 18, 540-548 (2016).

56. Kim, N. G. \& Gumbiner, B. M. Adhesion to fibronectin regulates Hippo signaling via the FAK-Src-PI3K pathway. J. Cell Biol. 210, 503-515 (2015)

57. Tang, Y. et al. MT1-MMP-dependent control of skeletal stem cell commitment via a beta1-integrin/YAP/TAZ signaling axis. Dev. Cell 25, 402-416 (2013).

58. Ohgushi, M., Minaguchi, M. \& Sasai, Y. Rho-signaling-directed YAP/TAZ activity underlies the long-term survival and expansion of human embryonic stem cells. Cell Stem Cell 17, 448-461 (2015).

59. Elosegui-Artola, A. et al. Force triggers YAP nuclear entry by regulating transport across nuclear pores. Cell 171, 1397-1410 e1314 (2017). 
60. Tharp, K. M. et al. Actomyosin-mediated tension orchestrates uncoupled respiration in adipose tissues. Cell Metab. 27, 602-615 e604 (2018).

61. Coats, B. R. et al. Metabolically activated adipose tissue macrophages perform detrimental and beneficial functions during diet-induced obesity. Cell Rep. 20, 3149-3161 (2017).

62. Weisberg, S. P. et al. Obesity is associated with macrophage accumulation in adipose tissue. J. Clin. Invest. 112, 1796-1808 (2003)

63. Cawthorn, W. P. \& Sethi, J. K. TNF-alpha and adipocyte biology. FEBS Lett. 582, 117-131 (2008).

64. Fain, J. N., Bahouth, S. W. \& Madan, A. K. TNFa release by the nonfat cells of human adipose tissue. Int J. Obes. 28, 616-622 (2004).

65. Hotamisligil, G. S., Shargill, N. S. \& Spiegelman, B. M. Adipose expression of tumor-necrosis-factor-alpha - direct role in obesity-linked insulin resistance. Science 259, 87-91 (1993).

66. Sabio, G. \& Davis, R. J. TNF and MAP kinase signalling pathways. Semin. Immunol. 26, 237-245 (2014).

67. Das, A., Fischer, R. S., Pan, D. \& Waterman, C. M. YAP nuclear localization in the absence of cell-cell contact is mediated by a filamentous actin-dependent, Myosin II- and phospho-YAP-independent pathway during extracellular matrix mechanosensing. J. Biol. Chem. 291, 6096-6110 (2016).

68. Strissel, K. J. et al. Adipocyte death, adipose tissue remodeling, and obesity complications. Diabetes 56, 2910-2918 (2007).

69. LeBlanc, L. et al. Yap1 safeguards mouse embryonic stem cells from excessive apoptosis during differentiation. Elife 7, e40167 (2018).

70. Vigneron, A. M., Ludwig, R. L. \& Vousden, K. H. Cytoplasmic ASPP1 inhibits apoptosis through the control of YAP. Genes Dev. 24, 2430-2439 (2010).

71. Kedan, A. et al. PYK2 negatively regulates the Hippo pathway in TNBC by stabilizing TAZ protein. Cell Death Dis. 9, 985 (2018).

72. Zhao, B. et al. TEAD mediates YAP-dependent gene induction and growth control. Genes Dev. 22, 1962-1971 (2008).

73. Farley, F. W., Soriano, P., Steffen, L. S. \& Dymecki, S. M. Widespread recombinase expression using FLPeR (flipper) mice. Genesis 28, 106-110 (2000).

74. Fang, J. et al. Sirt7 promotes adipogenesis in the mouse by inhibiting autocatalytic activation of Sirt1. Proc. Natl Acad. Sci. USA 114, E8352-E8361 (2017).

75. Bourgeois, F., Alexiu, A. \& Lemonnier, D. Dietary-induced obesity - effect of dietary fats on adipose-tissue cellularity in mice. Br. J. Nutr. 49, 17-26 (1983).

76. Berry, R. et al. Imaging of adipose tissue. Methods Enzymol. 537, 47-73 (2014).

77. Davis, M. P. A., van Dongen, S., Abreu-Goodger, C., Bartonicek, N. \& Enright, A. J. Kraken: A set of tools for quality control and analysis of high-throughput sequence data. Methods 63, 41-49 (2013).

78. Dobin, A. et al. STAR: ultrafast universal RNA-seq aligner. Bioinformatics 29, 15-21 (2013)

79. Liao, Y., Smyth, G. K. \& Shi, W. featureCounts: an efficient general purpose program for assigning sequence reads to genomic features. Bioinformatics 30, 923-930 (2014).

80. Love, M. I., Huber, W. \& Anders, S. Moderated estimation of fold change and dispersion for RNA-seq data with DESeq2. Genome Biol. 15, 550 (2014).

81. Apweiler, R. Activities at the Universal Protein Resource (UniProt) (vol 42, pg D198, 2014). Nucleic Acids Res. 42, 7486-7486 (2014).

82. Haim, Y., Tarnovscki, T., Bashari, D. \& Rudich, A. A chromatin immunoprecipitation (ChIP) protocol for use in whole human adipose tissue. Am. J. Physiol. Endocrinol. Metab. 305, E1172-E1177 (2013).

83. Polak, P. et al. Adipose-specific knockout of raptor results in lean mice with enhanced mitochondrial respiration. Cell Metab. 8, 399-410 (2008).

\section{Acknowledgements}

We thank Svea Hümmer for excellent secretarial help and Dagmar Magalei, Claudia Ullmann, Kathrin Heil, and Carola Meyer for technical assistance. This work is supported by the Cardio-Pulmonary Institute (CPI), EXC 2026, Project ID: 390649896, the National Natural Science Foundation of China (grant \#81870220) and Shaanxi Natural Science Fund for Distinguished Young Scholars of China (S2020-JCJQ-0239).

\section{Author contributions}

L.W. initiated and designed the study, performed most of the in vitro and in vivo experiments, analyzed data, and contributed to writing of the manuscript. S.P.W., Y.S., Z.Y., and E.L. carried out experiments with human adipocytes. S.P.W. performed the adenovirus Bim shRNA transduction experiments and discussed data. R.L. helped with in vivo experiments. Y.T.O. and M.P. helped with in vitro experiments. S.G. performed RNA sequencing and bioinformatics analysis. S.O. initiated, designed, and supervised the study, discussed data, and wrote the manuscript. All authors commented on the manuscript.

\section{Funding}

Open Access funding enabled and organized by Projekt DEAL.

\section{Competing interests}

The authors declare no competing interests.

\section{Additional information}

Supplementary information is available for this paper at https://doi.org/10.1038/s41467020-19229-3.

Correspondence and requests for materials should be addressed to L.W., S.W. or S.O.

Peer review information Nature Communications thanks the anonymous reviewers for their contribution to the peer review of this work.

Reprints and permission information is available at http://www.nature.com/reprints

Publisher's note Springer Nature remains neutral with regard to jurisdictional claims in published maps and institutional affiliations.

Open Access This article is licensed under a Creative Commons Attribution 4.0 International License, which permits use, sharing, adaptation, distribution and reproduction in any medium or format, as long as you give appropriate credit to the original author(s) and the source, provide a link to the Creative Commons license, and indicate if changes were made. The images or other third party material in this article are included in the article's Creative Commons license, unless indicated otherwise in a credit line to the material. If material is not included in the article's Creative Commons license and your intended use is not permitted by statutory regulation or exceeds the permitted use, you will need to obtain permission directly from the copyright holder. To view a copy of this license, visit http://creativecommons.org/ licenses/by/4.0/

(c) The Author(s) 2020 